\title{
1 Host-dependent fungus-fungus competition suppresses fungal pathogenesis in Arabidopsis
}

\section{2 thaliana}

4 Kuldanai Pathompitaknukul ${ }^{1,5}$, Kei Hiruma ${ }^{1,2,5^{*}}$, Hiroyuki Tanaka ${ }^{3,5}$, Nanami Kawamura ${ }^{1}$,

5 Atsushi Toyoda ${ }^{4}$, Takehiko Itoh $^{3}$, Yusuke Saijo $^{1 *}$

7 1. Department of Science and Technology, Nara Institute of Science and Technology, Nara

8 630-0192, Japan

9 2. PRESTO, Japan Science and Technology Agency, 4-1-8 Honcho Kawaguchi, Saitama 10 332-0012, Japan

11 3. Department of Biological Information, Tokyo Institute of Technology, Tokyo 152-8550, 12 Japan

13 4. National Institute of Genetics, Shizuoka 411-8540, Japan

14 5. These authors contribute equally.

15 *To correspondence; hiruma@bs.naist.jp and saijo@bs.naist.jp

\section{Abstract (149 words)}

18 Like animals, plants accommodate a rich diversity of microbes, typically without discernible 19 disease symptoms. How their pathogenesis is prevented in the host remains obscure. Here, we 20 show that the root-infecting fungus Colletotrichum fructicola of the C. gloeosporioides clade 21 (CgE), isolated from field-grown healthy Brassicaceae plants, inhibits growth of pathogenic 22 fungi in Arabidopsis thaliana, in a phosphate status-dependent manner. Loss of host ethylene 23 signaling or phytoalexins, camalexin or indole glucosinolates, however, allows CgE to display 24 pathogenesis, suggesting host contributions to endophytic $\mathrm{CgE}$ colonization and benefit. 25 Compared to a closely-related C. gloeosporioides pathogen $(\mathrm{CgP}), \mathrm{CgE}$ is characterized by 26 genome expansion and $>700$ fungal genes (4.34\%) specifically induced in the host roots when 27 co-inoculated with $\mathrm{CgP}$, including genes related to fungal secondary metabolism. This may 28 underlie antimicrobial tolerance of $\mathrm{CgE}$ and its dominance over pathogenic fungi within the 29 host, pointing to a role for fungus-fungus competition in asymptomatic fungal colonization in 30 plants.

\section{Keywords}


33 Fungal endophytes, fungal pathogenesis, microbe-microbe competition, secreted proteins,

34 methyltransferase, asymptomatic growth

Introduction

37

38 In nature, plants are intimately associated with a rich diversity of microbial communities,

39 including commensal, beneficial and pathogenic microorganisms (Bulgarelli et al., 2013;

40 Lundberg et al., 2012; Duran et al., 2018; Toju et al., 2018). Plants often establish beneficial

41 interactions with mutualistic microbes under adverse conditions. Most knowledge regarding

42 mutualistic plant-microbe interactions has been obtained from several symbiosis models,

43 including $\mathrm{N}_{2}$-fixing rhizobacteria and arbuscular mycorrhizal fungi, which promote host

44 acquisition of nitrogen and phosphorus, respectively (Lugtenberg \& Kamilova, 2009; Bonfante

$45 \&$ Genre, 2010). Compared to these symbionts, despite their richness and diversity in root

46 ecosystems, much less is known about the eco-physiological functions for fungal endophytes

47 that colonize within living plants without causing diseases (Rodriguez, 2009).

48 Beneficial functions of fungal endophytes include plant protection from pathogens (Gao et al.,

49 2010; Zhang et al., 2014). Direct protection relies on microbe-microbe competition between

50 endophytes and pathogens, often with antifungal compounds (Zivkovic et al., 2010).

51 Trichodema harzianum and Serendipita vermifera endophytes act as parasites to infect and

52 suppress phytopathogens, thereby conferring host protection (Druzhinina et al., 2011;

53 Moran-Diez et al., 2012; Sarkar et al., 2019). Species in Trichoderma genus also inhibit other

54 fungi with antifungal secondary metabolites (Schuster \& Schmoll, 2010). Many fungal toxins

55 can be produced in vitro without microbial competitors or hosts (Gao et al., 2010; Kunzler,

56 2018), whereas a few of them specifically require microbial competitors (Konig et al., 2013).

57 Conversely, some fungi employ ATP-binding cassettes and major facilitator superfamily

58 transporters to detoxify or export fungal toxins (Morrissey \& Osbourn, 1999; Gulshan \&

59 Moye-Rowley, 2007; Prasad \& Goffeau, 2012; Ruocco et al., 2009). These attacking and

60 defense mechanisms are likely to facilitate fungal competition with other microorganisms in the

61 common host (Abdullah et al., 2017; Stroe et al., 2020). Whether and if so how host plants

62 influence or exploit microbe-microbe competitions remain underexplored to date.

63 Beneficial bacteria and fungi also indirectly protect hosts by increasing local and/or 64 systemic pathogen resistance (Van Wees et al., 2008; Pieterse et al., 2014; de Lamo \& Takken, 65 2020). The endophytic ascomycete fungus Harpophora oryzae confers local and systemic rice 
resistance to rice blast fungi (Magnaporthe oryzae) (Xu et al., 2014). The basidiomycete Serendipita indica (formerly known as Piriformospora indica) induces systemic resistance in Arabidopsis thaliana against biotrophic powdery mildew, through the phytohormone jasmonic acid (JA) (Stein et al., 2008). However, molecular dissection of plant protection conferred by endophytic fungi has been hindered, in part due to the scarcity for genetic fungal studies in model plant species.

The ascomycete genus Colletotrichum causes anthracnose diseases in a wide range of crops, and is among the top 10 fungal pathogens of economic importance (Dean et al., 2012). Many Colletotrichum species are hemibiotrophic pathogens, displaying initial biotrophic and subsequent destructive necrotrophic phases (Perfect et al., 1999). In contrast to genuine obligate biotrophs such as powdery mildew and arbuscular mycorrhizal fungi, hemibiotrophic Colletotrichum species are amenable to axenic culture and genetic manipulation. In addition, high-quality genome sequences of over 10 species facilitate comparative genomics and molecular genetic studies in this genus (O'Connell et al., 2012; Gan et al., 2013 and 2016; Hacquard et al., 2016).

Colletotrichum genus has also endophytic species beneficial for the host plants. C. tofieldiae asymptomatically colonizes the roots of Arabidopsis thaliana, to promote plant growth under low-phosphate conditions. At the genome level, $C t$ is very closely related to root-infecting pathogenic species, such as $C$. incanum (Ci; Hacquard et al., 2016). Indeed, even $C t$ displays high virulence in the host plants lacking tryptophan (Trp)-derived antimicrobial metabolites (Hiruma et al., 2016). Ct overgrows and fails to promote plant growth in plants lacking MYB-type transcription factors $P H R 1$ and $P H L 1$, two major regulators of phosphate starvation responses (PSR) (Hiruma et al., 2016). PSR enhances phosphate uptake and utilization under phosphate deficiency by reprogramming root system architecture and gene expression (Bustos et al., 2010), but how PSR serves to prevent fungal overgrowth remains obscure. High relatedness between beneficial and pathogenic species seems to be widespread, rather than exceptional, in plant-inhabiting fungi (Rodriguez et al., 2009). Pathogenic species/strains are often found, without displaying virulence, in microbial communities on apparently healthy plants (García et al., 2012; Xu et al., 2014). In Arabidopsis thaliana, root-inhabiting bacteria may contribute to asymptomatic accommodation of filamentous microbial eukaryotes, by antagonizing their negative impacts on the host (Duran et al., 2018). How potential virulence of pathogens or commensals is suppressed to achieve asymptomatic accommodation represents an important question in both plants and animals (Hiruma et al., 2018). 
Here, we report an as-yet-undocumented beneficial Colletotrichum fungus, as well as its pathogenic relative, isolated from healthy field-grown cruciferous vegetables. Its colonization protects Arabidopsis thaliana plants from root-infecting fungal pathogens, in a manner dependent on ethylene, PSR and Trp-derived metabolites of the host. Transcriptional profiling in co-inoculated roots has produced an inventory of fungal genes that are specifically up- or down-regulated in the host-fungus-fungus interactions. Interestingly, both fungi strongly induce fungal genes related to fungal secondary metabolism. This implies chemical fungus-fungus competition dependent on the host, ultimately leading to suppression of fungal pathogenesis in plants.

\section{Results}

\section{Isolation of endophytic and pathogenic Colletotrichum fungi from field-grown}

\section{Brassicaceae vegetables}

We have assembled a total of 116 fungal isolates from the asymptomatic roots and/or leaves of Brassica spp. after surface disinfection. Of them, we selected ten isolates for further analysis, based on the ease in cultivation and morphological and growth characteristics in culture, which were reminiscent of previously described fungal endophytes. We assessed inoculation effects of these fungi on Arabidopsis thaliana plants following fungal hypha inoculation in $1 / 2 \times \mathrm{MS}$ agarose media. Twenty-one $\mathrm{d}$ after individual inoculation, we detected varied effects among the tested strains, ranging from plant growth promotion to inhibition, indicated by shoot fresh weight (SFW) (Supplementary Fig. 1A and B). In particular, inoculation with fungal isolates E35, E41 and E66 increased SFW under nutrient-sufficient conditions, on average, by 89\%, $51 \%$ and $115 \%$, respectively, while in contrast E40 inoculation drastically reduced plant SFW by $68 \%$, compared to mock controls. The results validate that healthy plants accommodate both plant growth-promoting (PGP) and pathogenic fungi.

Despite opposing effects on plant growth, pathogenic E40 and endophytic E41 fungi showed similar colony morphologies on potato dextrose agar (PDA) media, both characteristic of the Colletotrichum genus (Supplementary Fig. 1A). DNA sequencing of nuclear ribosomal internal transcribed spacer (ITS) regions, a universal DNA marker for fungal classification (Schoch et al., 2012), indicated that the two fungi were closely related to each other, within the clade Colletotrichum gloeosporioides (Supplementary Table 1), which we designated $C$. 
131 gloeosporioides pathogen $(\mathrm{CgP})$ and C. gloeosporioides endophyte (CgE), respectively.

132 Isolation of both fungi from apparently healthy plants prompted us to test the possible

133 involvement of endophytes in suppression of $\mathrm{CgP}$ pathogenesis in the host.

Endophytic CgE protects plants from pathogenic fungi

136 To examine what role CgE plays in host protection against pathogens, we co-inoculated

$137 \mathrm{CgP}$ and $\mathrm{CgE}$ spores onto Arabidopsis thaliana roots. Inoculation of $\mathrm{CgP}$ alone resulted in 138 severe inhibition of plant growth, indicated by a great decrease in SFW $21 \mathrm{~d}$ post inoculation 139 (dpi) (Fig. 1A and B). By contrast, no discernible disease symptoms were observed when 140 inoculated with $\mathrm{CgE}$ alone. Inoculation with $\mathrm{CgE}$ hyphae even promoted plant growth 141 (Supplementary Fig.1). Importantly, $\mathrm{CgE}$ co-inoculation with $\mathrm{CgP}$ significantly reduced disease 142 symptoms (Fig. 1A and B), compared with CgP inoculation alone. By contrast, co-inoculation 143 of heat-killed $\mathrm{CgE}$ spores did not affect $\mathrm{CgP}$ infection (Fig.1B). These results indicate that live $144 \mathrm{CgE}$ fungi are required for host protection from $\mathrm{CgP}$ in Arabidopsis thaliana. We validated 145 effectiveness of $\mathrm{CgE}$-mediated protection against another root-infecting pathogenic species, $C$. 146 incanum (Ci) (Sato et al., 2005; Hiruma et al., 2016; Hacquard et al., 2016), which is distantly 147 related to $\mathrm{CgP}$ (Supplementary Fig. 2). These results suggest that CgE protection exceeds beyond niche competition within the Colletotrichum gloeosporioides species complex.

CgE genome is characterized by long AT blocks with potential in generating SSP diversity

We obtained whole-genome information for $\mathrm{CgE}$ and $\mathrm{CgP}$. Whole-genome alignment indicated $\mathrm{CgE}$ and $\mathrm{CgP}$ as different species in related taxa of the Colletotrichum gloeosporioides species complex (Fig. 1C). CgP genome size was approximately $57 \mathrm{Mb}$, similar to that of $C$. fructicola (previously described as C. gloeosporioides) Nara gc-5 strain (55.6 Mb, Gan et al., 2013), while CgE genome size was approximately $64.5 \mathrm{Mb}$, far larger compared to the other $\mathrm{Cg}$ strains sequenced to date $(53.2-57.7 \mathrm{Mb}$ ) (Supplementary Fig. 3). Genome comparison revealed large AT-rich regions ( $\mathrm{GC}$ content $<40 \%$ ) as a unique feature of $\mathrm{CgE}$ genome, which largely explain increased genome size (Supplementary Fig. 4). Repeat-induced point mutations (RIP) protect ascomycete fungal genomes against transposable elements, by converting C-G base pairs to T-A in duplicated sequences (Galagan \& Selker, 2004). RIP indices (TpA/ApT dinucleotide ratios) were high in the AT-rich regions, in both genomes, (Fig. 
162 1D), consistent with their generation by RIP. A specific feature of $\mathrm{CgE}$, not conserved in $\mathrm{CgP}$, 163 included the existence of 13 genes predicted in AT-rich regions, which were all located near the 164 borders with GC-rich regions (GC content $>40 \%$ ). A border gene, CGE00232, appears to be 165 generated via insertion of an AT-rich region into a conserved syntenic gene in CgP (Fig.1D). In 166 GC-rich regions, both genomes had gaps at non-syntenic positions, at a considerably high 167 frequency (Supplementary Fig. 6). Clustering protein-coding sequences into sets of orthologous 168 genes with Proteinortho revealed that, of $15,763 \mathrm{CgE}$ and $14,830 \mathrm{CgP}$ gene families in total, 16913,331 gene families were shared by the two fungi, while 2432 and 1499 gene families were 170 specific to $\mathrm{CgE}$ and $\mathrm{CgP}$, respectively (Fig.1E; Supplementary Table 2). These results indicate 171 that the two genomes are more diverged than expected from the ITS sequences.

172 Fungal biotrophy relies on small secreted proteins (SSPs), which, if not all, contribute to 173 suppression of host immunity (O'Connell et al., 2012; Lo Presti et al., 2015). The gene number 174 of predicted SSPs was far the greatest in CgE among the sequenced Cg strains (Supplementary 175 Table 2), despite similarity in the number of cell wall degrading enzymes (CWDEs), 176 transporters, cytochrome P450 and secondary metabolite clusters (Supplementary Table 3, 4, 5,

177 6, and 7). This points to specific expansion of a SSP repertoire in CgE, in agreement with less 178 destructive mode of infection.

\section{$\mathrm{CgE}$ inhibition of $\mathrm{CgP}$ growth is host dependent}

We next tested whether CgE inhibition of pathogen growth occurs without the host, on a dual culture plate with $\mathrm{CgE}$ and $\mathrm{CgP}$ following inoculation onto the opposite sides. Although antibiotic Hygromycin B (100 $\mu \mathrm{M})$ eventually suppressed colony growth of both fungi, CgE showed higher Hygromycin tolerance than CgP (Fig. 2A). By contrast, when co-cultured, CgP growth was not inhibited on the $\mathrm{CgE}$ side, suggesting that $\mathrm{CgE}$ does not directly inhibit $\mathrm{CgP}$ growth at least under the tested culture conditions.

187 We then tested whether CgE restricts CgP growth in-planta, by quantitative PCR analysis 188 with fungal species-specific primers (Fig.2B, Supplementary Table 13). CgP growth was greatly reduced $3 \mathrm{~d}$ post-inoculation (dpi) when co-inoculated with $\mathrm{CgE}$, compared to $\mathrm{CgP}$ inoculation alone, while $\mathrm{CgE}$ growth was not affected by $\mathrm{CgP}$ (Fig. 2B). These results suggest that $\mathrm{CgE}$ outcompetes $\mathrm{CgP}$ in the host roots. We also employed transgenic $\mathrm{CgP}$ fungi constitutively expressing green fluorescence protein (CgP-GFP) under the control of GPDA regulatory DNA 
sequences from Aspergillus nidulans (O'Connell et al., 2007). Following co-inoculation of CgP-GFP with $\mathrm{CgE}$, we traced live $\mathrm{CgP}$ growth and determined its abundance with the GFP signal as a proxy. Live imaging revealed that hyphal network of CgP-GFP in the roots was much less developed at $3 \mathrm{dpi}$ in the presence of $\mathrm{CgE}$ than in its absence (Fig. 2C), suggesting that $\mathrm{CgE}$ restricts $\mathrm{CgP}$ hyphal growth at an early infection stage. In the absence of $\mathrm{CgE}, \mathrm{CgP}$ produced new GFP-positive spores even at 3 dpi (Fig. 2C), and then formed numerous melanized structures at 10 dpi (Fig. 2D). These results suggest that $\mathrm{CgE}$ colonization inhibits growth and reproduction of $\mathrm{CgP}$ in Arabidopsis thaliana roots.

\section{Endophytic CgE colonization and host protection are phosphate status dependent}

C. tofieldiae was reported to promote plant growth, specifically under phosphate deficiency in a manner dependent on the major PSR-regulating transcription factors PHR1/PHL1 (Hiruma et al., 2016). In phr1 phl1 plants, C. tofieldiae overgrows and fails to confer plant growth promotion, implying a role for PHR1/PHL1 in suppression of potential fungal pathogenesis. We tested possible phosphate status dependence of beneficial $\mathrm{CgE}$ interaction, in co-inoculation assays under normal $\left(625 \mu \mathrm{M} \mathrm{KK_{2 }} \mathrm{PO}_{4}\right)$ and low phosphate $\left(50 \mu \mathrm{M} \mathrm{KK_{2 }} \mathrm{PO}_{4}\right)$ conditions. $\mathrm{CgP}$ caused severe diseases irrespective of phosphate conditions (Figs 3A and B). Surprisingly, CgE also caused disease symptoms under low phosphate conditions, albeit to a lesser degree than $\mathrm{CgP}$, and no longer protected the host despite slight alleviation of $\mathrm{CgP}$ pathogenesis (Figs 3A and $\mathrm{B})$. These results suggest that $\mathrm{CgE}$ becomes pathogenic when phosphate is limited, in contrast to C. tofieldiae. Nevertheless, $\mathrm{CgE}$ disease symptoms became more severe in phrl phl1 plants, pointing to a critical role for $P H R 1 / P H L 1$ in restricting fungal pathogenesis under phosphate deficiency for both $\mathrm{CgE}$ and C. tofieldiae (Fig. 3C).

\section{Endophytic CgE colonization and host protection require plant ethylene signaling}

Ethylene, JA, and salicylic acid (SA) are among the major defense-related hormones that greatly influence plant-microbe interactions (Robert-Seilaniantz et al., 2011; Pieterse et al., 2012). To determine the possible involvement of these hormone pathway(s) in beneficial interactions with $\mathrm{CgE}$, we tested whether and if so how fungal infection modes and plant growth are influenced when the master regulator of ethylene signaling EIN2, enzymes required for JA and SA biosynthesis, DDE2 and SID2, respectively, and SA signaling regulator PAD4 are mutated. In ein 2 pad4 sid2 and dde2 ein 2 sid2 plants, $\mathrm{CgE}$ inoculation or co-inoculation with $\mathrm{CgP}$ resulted in severe growth retardation, pointing to a critical role for ethylene signaling in 
226 endophytic CgE colonization. By contrast, dde2 pad4 sid2 plants largely retained WT-like

227 growth and acquired $\mathrm{CgP}$ resistance after $\mathrm{CgE}$ inoculation (Supplementary Fig. 6A). These data

228 suggest a pivotal role for host ethylene in the endophytic colonization and host-protective

229 function of $\mathrm{CgE}$.

230 We validated this notion in different ethylene-related mutants. ein2-1 plants were 231 hyper-susceptible to $\mathrm{CgE}$, and were not protected by $\mathrm{CgE}$ against $\mathrm{CgP}$ (Fig. $4 \mathrm{~A}$ and B). ein 3 and 232 eill plants, lacking ethylene-related transcription factors EIN3 or EIL1, respectively (An et al., 233 2010), also displayed disease-like symptoms when inoculated with CgE (Fig. 4A and B, 234 Supplementary Fig. 6B).

236 Endophytic CgE colonization and host protection require host tryptophan (Trp)-derived 237 metabolites

238 In Arabidopsis thaliana, Trp-derived secondary metabolites are required for the proper 239 control of both pathogenic and endophytic fungi (Bednarek et al., 2009; Hiruma et al., 2016).

240 As expected, loss of cytochrome P450-mediated conversion of Trp to indole-3-acetaldoxime, 241 the initial catalytic step in this pathway (Fig. 5A), rendered cyp79B2 cyp79B3 plants 242 super-susceptible to $\mathrm{CgP}$ and also succumbed to $\mathrm{CgE}$, allowing its pathogenesis (Fig. 5B).

243 Disruption of PENETRATION2 (PEN2) atypical myrosinase (Lipka et al., 2005; Bednarek et 244 al., 2009) or PHYTOALEXIN DEFICIENT 3 (PAD3) cytochrome P450 monooxygenase 245 CYP71B5 required for antifungal camalexin biosynthesis (Zhou et al, 1999) also lost the control 246 of $\mathrm{CgE}$ colonization and plant protection (Figs. 5A and 5B), as described for C. tofieldiae 247 (Hiruma et al., 2016). As expected, pen 2 and pad3 plants were both more susceptible to CgP 248 than WT plants. These results indicate that potential virulence of $\mathrm{CgE}$ is de-repressed in the 249 absence of host Trp-derived antimicrobial metabolites, and that its suppression is a key for beneficial interactions with $\mathrm{CgE}$.

251 We then examined whether exogenous application of synthesized Trp-derived metabolites 252 inhibits fungal growth in culture. In the presence of camalexin and indole-3-carbinol (I3C), 253 growth of $\mathrm{CgE}$ and $\mathrm{CgP}$ was both suppressed, indicated by the colony diameters (Fig.5A, 254 Supplementary Figs. 7A and 7B). By contrast, indole-3-ylmethylamine (I3A) did not suppress 255 either growth (Supplementary Figs. 7A and 7B). These results suggest that specific subsets of 256 Trp-derived antifungal metabolites (Camalexin, I3C and PEN2-dependent compounds 257 excluding I3A, Fig. 5A) directly attenuate fungal growth to establish an endophytic mode in 
$258 \mathrm{CgE}$. Interestingly, $\mathrm{CgE}$ again showed greater tolerance than $\mathrm{CgP}$ to camalexin and $\mathrm{I} 3 \mathrm{C}$ in 259 culture (Supplementary Figs. 7A and 7B), highlighting CgE tolerance to antifungal metabolites.

260 This implies CgE adaptation to the root interior in Arabidopsis thaliana, wherein antifungal

261 Trp-derived metabolites are highly induced in response to fungal challenge.

262

263 Host transcriptome is not greatly altered during $\mathrm{CgE}$ colonization or protection

264 To have an overview of the host responses during CgE protection, we conducted RNA 265 sequencing analysis in $\mathrm{CgE}-, \mathrm{CgP}-$, and co-inoculated roots at $6 \mathrm{~h}$ and $3 \mathrm{dpi}$. We first compared 266 overall transcriptome profiles in multidimensional scaling analysis (Supplementary Fig. 8A).

267 Although the host transcriptomes were not clearly separated between different inoculums and 268 mock control at 6 hpi, fungal inoculation effects became apparent at 3 dpi (Supplementary Fig. 269 8A), implying intensive fungal challenge and/or host defense activation at this stage. 270 Importantly, $\mathrm{CgE}$ and $\mathrm{CgP}$ inoculation differentially impacted the host transcriptome at $3 \mathrm{dpi}$ 271 (Supplementary Fig. 8A), consistent with striking differences in the host outcomes between the 272 two fungi (Fig. 1A). Of particular note, root transcriptomes were nearly indistinguishable 273 between $\mathrm{CgE}$ inoculation alone and co-inoculation with $\mathrm{CgP}$, but far different from that of $\mathrm{CgP}$ 274 inoculation alone, consistent with a collapse of $\mathrm{CgP}$ growth by $\mathrm{CgE}$ co-inoculation (Fig. 2B). $275 \mathrm{CgE}$ colonization essentially masked $\mathrm{CgP}$ effects on the host transcriptome.

276 Pairwise transcriptome comparisons [false discovery rate (FDR) $<0.01$ ] revealed 13,300 277 differentially expressed genes (DEGs) at least in one of the pairs compared. These DEGs were 278 classified into 12 different clusters by K-means clustering. Clusters 6, 11, and 12 were 279 characterized by genes strongly responsive to both fungi, with Gene Ontologies (GOs) 280 "response to chitin," "innate immune response," "Trp metabolism (tryptophan biosynthetic and 281 metabolic process, glucosinolate biosynthetic and metabolic process)," and "plant hormonal 282 response" dominating (Supplementary Fig. 8B, Supplementary Table 8). In clusters 11 and 12 , 283 in addition to defense responses, GOs related to hypoxia and ethylene signaling 284 (ethylene-activated signaling pathway, response to ethylene, cellular response to ethylene 285 stimulus) were overrepresented. Cluster 6, 11 and 12 were over-represented with genes 286 strongly induced in response to $\mathrm{CgP}$ (Supplementary Fig. 8B, Supplementary Table 8), 287 suggesting that $\mathrm{CgP}$ induces stronger defense activation than $\mathrm{CgE}$, at this early interaction stage. 288 Notably, this $\mathrm{CgP}$ effect was nearly abolished by $\mathrm{CgE}$ co-inoculation (Supplementary Fig. 8B), suggesting that host defense activation was alleviated in the presence of $\mathrm{CgE}$. 
$\mathrm{CgE}$ suppression of host transcriptional reprograming in response to $\mathrm{CgP}$ prompted us to examine fungal transcriptome during $\mathrm{CgE}$-mediated host protection. We assembled in-planta fungal transcriptomes by separating $\mathrm{CgE}$ - and $\mathrm{CgP}$-derived sequence reads in the co-inoculated roots, based on RNA-sequencing read mismatching to $\mathrm{CgE}$ and $\mathrm{CgP}$ genomes (Fig. 6A). Our method successfully identified the origin of the $93 \%$ sequence reads. Nearly a half of the total reads $(48.13 \% \pm 9.11 \%)$ were derived from $\mathrm{CgE}$, whereas only a small portion $(4.466 \% \pm$ $0.32 \%$ ) was derived from $\mathrm{CgP}$ (Supplementary Table 9). These results agree with $\mathrm{CgE}$ outcompeting over $\mathrm{CgP}$ (Fig. 2B and C).

Next, we focused on in-planta fungal DEGs $\left(\left|\log _{2} \mathrm{FC}\right|>1\right.$, FDR $\left.<0.05\right)$ between individually-inoculated and co-inoculated roots. We detected 892 and 1,239 DEGs in CgE and $\mathrm{CgP}$, respectively (Fig. 6A). Of the $721 \mathrm{CgE}$ DEGs up-regulated in response to $\mathrm{CgP}$, a large class were genes encoding SSPs, CWDEs (Supplementary note), secondary metabolite-related proteins including cytochrome $\mathrm{P} 450$, transporters, and antibiotic resistance proteins (Supplementary Table 10). For instance, of $659 \mathrm{CgE}$ genes annotated for SSPs, 47 and 27 genes were up- and down-regulated following $\mathrm{CgP}$ co-inoculation, respectively (Fig. 6B). Although the majority of these $\mathrm{CgE} S S P$ genes (67 genes $>90 \%$ ) were also conserved in $\mathrm{CgP}$, most of $\mathrm{CgP}$ homologous genes (49 genes $>70 \%$ ) displayed distinct expression patterns in the host (Fig. 6B). Of $618 S S P$ genes in $\mathrm{CgP}, 32$ and 82 genes were up- and down-regulated, respectively, following $\mathrm{CgE}$ co-inoculation. Of these $114 \mathrm{CgP}$ SSP genes, 104 genes were conserved in $\mathrm{CgE}$ genome but again displayed distinct expression patterns. These results suggest that the two fungi, despite close relatedness, express separate sets of SSPs during their competition in roots.

GO related to methylation (e.g., methyltransferases) dominated in $\mathrm{CgE}$ up-regulated genes

314 following CgP co-inoculation (GO:0032259, FDR: 9.8E-9, Fig. 6C). Notably, 44 out of 94

315 LaeA-like (LaeA and llm) methyltransferase genes (annotated as Secondary metabolism 316 regulator LAE1 or laeA) were highly induced in $\mathrm{CgE}$ during interaction with $\mathrm{CgP}$ in roots $\left(\log _{2}\right.$ 317 FC $>1$, FDR $<0.05$, Supplementary Table 11). In different fungi, their homologues regulate 318 production of secondary metabolites including fungal toxins (Palmer et al., 2013, 319 Supplementary Fig 9). In addition, several methyltransferase genes other than LaeA-like were 320 also highly induced during CgE-CgP competition. These data suggest the possible involvement 321 of fungal secondary metabolites in the fungus-fungus competition. Indeed, $\mathrm{CgE}$ genes related to 322 biosynthesis and efflux of secondary metabolites including fungal toxins, e.g., echinocandin B, $323 \mathrm{~T}-2$ toxin, botrydial, aspyridones, were over-represented in $\mathrm{CgP}$-inducible DEGs, in the host 
324 (Supplementary Table 10). Furthermore, genes encoding cytochrome P450 monooxygenase,

325 FAD-linked oxidoreductase, efflux pump, acyltransferase, prosolanapyrone synthase, C-factor, 326 transcription factor and (N- and O-) methyltransferases in a CgE-specific secondary metabolism 327 cluster (Cluster 25), closely located to an AT-rich region, were highly activated following CgP 328 co-inoculation (Fig. 6D, Supplementary Fig. 5, Supplementary Table 10). Our results imply that 329 CgE produces diverse secondary metabolites including fungal toxins in the host, in response to $330 \mathrm{CgP}$, thereby suppressing $\mathrm{CgP}$ growth. Conversely, $\mathrm{CgP}$ also seems to produce different sets of 331 fungal secondary metabolites in the host, in response to $\mathrm{CgE}$, indicated by activation of some of 332 LaeA-like methyltransferase genes (29 of 89 genes). Our results imply secondary 333 metabolite-based fungus-fungus competition in the host (Supplementary Fig. 10; Supplementary 334 Table 10 and 11).

335 Our in vitro culture assay revealed that $\mathrm{CgP}$ was more sensitive than $\mathrm{CgE}$, to the antifungal compound echinocandin $\mathrm{B}$, which inhibits synthesis of $\beta-(1,3)$-glucan (a major structural component of the fungal cell wall) (Walker et al., 2010, Supplementary Fig. 11), although the two fungi were essentially equally sensitive to another fungal toxin, aspyridone A (Macheleidt et al., 2016). Consistent with $\mathrm{CgE}$ tolerance to fungal toxins, an $\mathrm{ABC}$ transporter gene

340 (CGE02297) related to fungal toxin efflux was strongly activated in CgE (Supplementary Table 341 10). The lack for dramatic transcriptome-wide changes in the host (Supplementary Fig. 8) 342 implies that these toxins are specific to fungi (Walker et al., 2010). Our findings discover an 343 important role for fungus-fungus competition, possibly via fungal toxins, in host protection by 344 beneficial fungi.

\section{Discussion}

\section{Contrasting lifestyles of two closely related Colletotrichum fungi in the host roots}

In this study, we reveal two fungal species of the C. gloeosporioides clade, which is best known as devastating pathogens causing anthracnose diseases on important crops (Weir et al., 2012, Gan et al., 2013; Zhang et al., 2018). CgE is a root-associated endophyte conferring plant protection, whereas $\mathrm{CgP}$ is a highly virulent, root-infecting pathogen, both isolated from asymptomatic radish plants. Our results in C. gloeosporioides clade strengthen divergence of infection modes in Colletotrichum fungi, as described for $C$. spaethianum clade, including 
al., 2016). These findings are consistent with the view that fungal pathogens have independently diversified infection modes in separate fungal lineages (Raffaele \& Kamoun, 2012).

Increased genome size of $\mathrm{CgE}$ is largely explained by large AT-rich blocks. A similar case was reported between distantly related C. orbiculare 104-T and C. fructicola Nara gc5 (Fig.1A, Gan et al., 2013; 2019). Our evidence demonstrates genome expansion with AT-rich regions even within closely-related species of the same fungal clade. Such genome expansion is often associated with diversification of virulence factors such as SSPs or secondary metabolism-related genes in plant-infecting fungi (Rouxel et al., 2011). Of $13 \mathrm{CgE}$ genes located in AT-rich blocks, expression of 5 genes has been validated during root colonization (Supplementary Table 12). Notably, genes located adjacent to an AT-rich block (secondary metabolism cluster 25) were highly induced specifically during fungal competition in the host (Fig. 6D), consistent with a role for AT-rich blocks in transcriptional activation (Nishi \& Itoh, 1986; Palida et al., 1993). In Epichloë and Neotyphodium grass symbionts producing an extraordinarily diverse panel of anti-insect alkaloids, secondary metabolism clusters are present in proximity to AT-rich blocks (Schardl et al., 2013). AT-rich regions likely contribute to rapid evolution of part of microbial genomes, as illustrated in a "two-speed genome" model (Sanchez-Vallet et al., 2018), and stress-responsive gene regulation.

Comparative analysis for three different $\mathrm{Cg}$ genomes reveals that genes related to cell wall degradation, cytochrome P450 and secondary metabolite clusters are conserved in $\mathrm{CgE}$ and related pathogenic strains. Notably, however, SSP gene family is greatly expanded in CgE, of which some are specifically induced during competition with $\mathrm{CgP}$ in Arabidopsis thaliana roots (Fig. 6B, Supplementary Table 10). This is marked contrast to SSP repertoire in C. tofieldiae, which is reduced compared with closely related pathogenic species (Hacquard et al., 2016). Constraint of SSP repertoire in nonpathogenic relative to pathogenic species is also seen in Fusarium oxysporum (de Lamo et al., 2020). It is tempting to speculate that CgE utilizes SSPs to limit the opponent's growth, rather than to promote infection, in the host.

\section{Endophytic colonization of Colletotrichum fructicola requires host ethylene}

In Arabidopsis thaliana, $\mathrm{CgE} / \mathrm{CgP}$ co-inoculation results in suppression of $\mathrm{CgP}$ virulence, consistent with their asymptomatic colonization in Brassicaceae vegetables. This requires ethylene-dependent suppression of potential $\mathrm{CgE}$ virulence. Ethylene signaling leads to 
during pathogen resistance, in particular against necrotrophs (Thomma et al., 2001). Ethylene has also been implicated in beneficial interactions with mutualistic microbes (Zamioudis \& Pieterse, 2012). Fusarium oxysporum disease suppression by endophytic Fusarium solani in tomato also requires the host ethylene (Kavroulakis et al., 2007). However, it is not clear whether ethylene signaling contributes to endophytic (non-pathogenic) colonization of $F$. solani by suppressing necrotrophy. Our findings with $\mathrm{CgE}$ extend a role for ethylene in suppression of fungal necrotrophy to endophytic species.

Remarkably, C. tofieldiae-mediated plant growth promotion is specific to phosphate deficiency, whereas CgE-mediated protection is specific to phosphate sufficiency (Fig.3). This provides compelling evidence for nutrition-dependent shifting of mutualistic fungal benefits and partners in plants. Interestingly, despite opposing effects of phosphate status on host benefits, both fungi overgrow in phrl phll plants under phosphate deficiency, pointing to a critical role for PHR1/PHL1-mediated PSR in restriction of fungal growth and pathogenesis (Fig. 3). PSR positively influences EIN3 protein accumulation during root hair formation under low phosphate conditions (Song et al., 2016; Liu et al., 2017). Conversely, EIN3 and EIL1 positively regulate PHRI expression in response to ethylene (Liu et al., 2017). Mutual positive feedback regulations between ethylene and PSR signaling may underlie PHR1/PHL1-mediated suppression of potential $\mathrm{CgE}$ pathogenesis. Ethylene also restricts biotrophic colonization of arbuscular mycorrhiza under low-Pi conditions (Kloppholz et al., 2011). However, C. tofieldiae colonization and plant growth promotion are unaffected by dysfunction of ethylene signaling (Hiruma et al., 2016), pointing to an ethylene-independent fungal control via PHR1/PHL1. PHR1/PHL1 also negatively regulate SA-based defenses in assembling root-associated bacterial communities (Castrillo et al., 2017). How PHR1/PHL1 contribute to endophytic fungal colonization merits further in-depth studies.

\section{Fungus-fungus competition provides a basis for CgE-mediated host protection}

414 Host-dependent $\mathrm{CgE}-\mathrm{CgP}$ competition predicts the existence of a critical trigger for 415 anti-fungal mechanisms in $\mathrm{CgE}$, when it encounters a fungal competitor in the host. 416 Host-dependent, competitor-induced extensive reprogramming of fungal transcriptome (Fig.6,

417 Supplementary Fig. 9), without substantially affecting the host transcriptome (Supplementary 418 Fig. 8), implies fungus-specific and -inducible nature of $\mathrm{CgE}$ competition mechanisms. 419 Transcriptome data imply involvement of fungal secondary metabolites, including several 420 toxins, during fungus-fungus competition. Fungal toxins are often produced as secondary 
421 metabolites under adverse conditions to the fungi, e.g. during host infection or anti-microbial

422 defenses, and are often associated with fungal necrotrophy (Osbourn, 2010). Notably, however,

$423 \mathrm{CgE}$ specifically induces these genes in the host, without impeding plant growth, in response to

$424 \mathrm{CgP}$.

425 Nearly a half (46\%) of CgE LaeA-like methyltransferase genes are highly induced during 426 competition with $\mathrm{CgP}$ in roots. LaeA is a putative methyltransferase that modulates 427 heterochromatin structures (Bok \& Keller, 2004; Reyes-Dominguez et al., 2010; Palmer et al., 428 2013). Deletion of LaeA in several fungal species lowers production of fungal secondary 429 metabolites including fungal toxins, as well as fungal growth and virulence (Bok et al., 2006; 430 Bouhired et al., 2007; Kale et al., 2008; Lodeiro et al., 2009; Wiemann et al., 2009). 431 Conversely, $\mathrm{CgP}$ also induces different sets of fungal toxin-related genes and LaeA-like 432 methyltransferases in the host, in response to $\mathrm{CgE}$, albeit to a lesser degree compared with $\mathrm{CgE}$. 433 Increased numbers of LaeA-like methyltransferase genes in both $\mathrm{CgE}$ and $\mathrm{CgP}$ (94 and 86 genes, 434 respectively) are notable compared to saprotrophic Aspergillus nidulans (10 genes) (Palmer et 435 al., 2013). Repertoire expansion of fungal secondary metabolite regulators and their induction 436 during host colonization in response to another fungus, suggests a critical role for fungal 437 secondary metabolites in fungus-fungus competition. Acquisition of a fungal toxin-detoxifying 438 enzyme gene of endophyte origin in wheat confers Fusarium head blight resistance (Wang et al., 439 2020). In bacteria, type IV and VI secreted systems are employed to directly inject toxins to 440 eukaryotic and bacterial competitors (Basler et al., 2013; Ma et al., 2014; Souza et al., 2015; 441 Trunk et al., 2018; Kim et al., 2019). Interestingly, Pseudomonas aeruginosa appears to sense 442 the presence of functional type VI secretion in Vibrio cholerae competitor (Basler et al., 2013).

443 These studies and ours suggest the existence of mechanisms by which infectious microbes 444 respond to competitors in the host environment. How the host influences microbe-microbe 445 competitions for host benefits merits future studies.

447 Materials and methods

449 Plant-fungus interaction assay by plant and fungal cocultures

$450 \mathrm{CgE}$ and $\mathrm{CgP}$ were mainly used for plant-fungus interaction assay. In brief, 7-day-old plants 451 grown on $1 / 2 \mathrm{MS}$ media with $25 \mathrm{mM}$ sucrose were placed to $1 / 2 \mathrm{MS}$ media without sucrose in 
453 onto the plant root tips ( $5 \mu \mathrm{l}$ each plant). The initial spore suspension of $\mathrm{CgE}$ and $\mathrm{CgP}$ in each

454 treatment was adjusted to the same amount ( 25 spores/plant). The mixed suspension contained

455 the same amount of $\mathrm{CgE}$ and $\mathrm{CgP}$ spores (each 25 spores/plant). Dead spores were prepared by

456 autoclaving $\left(121^{\circ} \mathrm{C}, 15 \mathrm{~min}\right)$. Plates were placed horizontally in a temperature-controlled room

457 with a photoperiod of 12 -h light/12-h dark and temperature of $21^{\circ} \mathrm{C} \pm 1{ }^{\circ} \mathrm{C}$. Full details are given

458 in Supplementary Experimental Procedures.

459

460 Fluorescence microscopy

461 Inoculated Arabidopsis thaliana roots were visualized using fluorescence microscopy. The 462 studies were performed using a confocal laser scanning microscope Olympus FV1000 with 463 excitation at $488 \mathrm{~nm}$ for GFP or bright field and $560 \mathrm{~nm}$ for propidium iodide (PI) at 10x, 20x, 464 and $40 \mathrm{x}$ magnification. PI $(10 \mathrm{mg} / \mathrm{ml})$ was used to stain root cell walls by direct application onto the slide.

466

\section{Genome sequencing and assembly}

468 Fungal DNA was extracted by CTAB with RNase treatment from fungal hyphae grown on 469 liquid Mathur's medium (glucose $2.8 \mathrm{~g} / 1, \mathrm{MgSO}_{4} \cdot 7 \mathrm{H}_{2} \mathrm{O} 1.2 \mathrm{~g} / \mathrm{l}, \quad \mathrm{KH}_{2} \mathrm{PO}_{4} 2.7 \mathrm{~g} / \mathrm{l}$, and 470 mycological peptone LP0040 $2.2 \mathrm{~g} / \mathrm{l}$ ) for 2 days. Genomic sequences of $\mathrm{CgE}$ and $\mathrm{CgP}$ were 471 determined using PacBio single-molecule real-time sequencing and Illumina HiSeq for 472 paired-end short reads. Genome sequences for $\mathrm{CgE}$ and $\mathrm{CgP}$ are deposited in DDBJ 473 (DRA009690, CgE=CfE). Full details about genome sequencing, genome assembly, gene 474 prediction, gene annotation and comparative genomics are given in Supplemental Experimental 475 Procedures.

\section{Transcriptome analysis}

478 RNA samples were extracted from inoculated roots at post-inoculation $6 \mathrm{~h}$ and 3 dpi. Total 479 RNA was extracted using a NucleoSpin RNA Plant (Macherey-Nagel). RNA samples (1 $\mu \mathrm{g}$ 480 each) were then sent to Macrogen for library preparation and subsequent sequencing. RNA-seq 481 read sets obtained from $\mathrm{CgE}, \mathrm{CgP}$, and co-inoculated samples were subjected to adapter 482 removal and quality filtering using Platanus_trim (version 1.0.7) with default parameters. The 483 trimmed reads were classified using two sequential rounds of mapping. First, the trimmed reads 484 were mapped to the Arabidopsis thaliana genome using HISAT (version 2.1.0) (Kim et al., 
485 2015) with default parameters. Reads that were mapped onto the Arabidopsis thaliana genome

486 were classified as originating from Arabidopsis thaliana. Next, we performed the second

487 classification by mapping the reads that remained unmapped in the first classification onto $\mathrm{CgE}$

488 and $\mathrm{CgP}$ genomes. Reads that uniquely mapped to either $\mathrm{CgE}$ or $\mathrm{CgP}$ genomes were classified

489 as originating from $\mathrm{CgE}$ or $\mathrm{CgP}$, respectively. In addition, reads that could not be mapped to

490 both genomes were classified as unmapped reads. Finally, reads that mapped to both genomes

491 during the second step were further classified. The number of mismatches in the alignment

492 reads were identified by the "XM" flag in the SAM output files and then the number of

493 mismatches in the alignment against $\mathrm{CgE}$ and $\mathrm{CgP}$ genomes were compared. Based on the

494 comparison results, reads were classified into $\mathrm{CgE}, \mathrm{CgP}$, and classified reads. The resulting

495 CgE-RNA-seq derived $\mathrm{CgE}$ reads (CgE-CgE reads), CgP-RNA-seq derived $\mathrm{CgP}$ reads

496 (CgP-CgP reads), co-inoculation-RNA-seq derived $\mathrm{CgE}$ reads (coinoc-CgE reads), and

497 co-inoculation-RNA-seq derived $\mathrm{CgP}$ reads (coinoc-CgP reads) were used for differential

498 expression analysis. RNAseq sequences used in this study are deposited in DDBJ (DRA009854,

$499 \mathrm{CgE}=\mathrm{CfE})$. Full details are given in Supplementary Experimental Procedures.

500

501 Supplementary information includes Supplementary Experimental Procedures, Supplementary note, 11 figures, and 14 table.

503

\section{Contributions}

$505 \mathrm{KH}$, YS conceived the study. PK, KH, HT, NK, and AT conducted the experiments. PK, KH, 506 HT, NK, AT and TI analyzed the data. PK, KH, and YS wrote the paper with feedback from all 507 the co-authors.

508

\section{Acknowledgements}

510 We thank Ms. Mie Matsubara, and Ms. Akemi Uchiyama for technical assistance, and Dr.

511 Shigetaka Yasuda for advice on fungal growth assays with tryptophan-derived metabolites. We

512 thank Dr. Yasuyuki Kubo for Agrobacterium C58C1 strains. PK was supported from The

513 NAIST International Scholarship. This work was supported in part by Japan Society for the

514 Promotion of Sciences (JSPS) KAKENHI Grant (16H06279, 16KT0031, 18H04822, 18K14466,

515 18H02467), the Japan Science and Technology Agency (JST) grant (JPMJPR16Q7,

516 JPMJSC1702), and Asashi Grass Foundation. Computations were partially performed on the

517 NIG supercomputer at ROIS National Institute of Genetics. 
519 References

5201 Abdullah, A. S. et al. Host-Multi-Pathogen Warfare: Pathogen Interactions in

521 Co-infected Plants. Front Plant Sci 8, 1806, doi:10.3389/fpls.2017.01806 (2017).

5222 Alonso, J. M., Hirayama, T., Roman, G., Nourizadeh, S. \& Ecker, J. R. EIN2, a

523 bifunctional transducer of ethylene and stress responses in Arabidopsis. Science 284, 2148-2152,

524 doi:DOI 10.1126/science.284.5423.2148 (1999).

5253 An, F. Y. et al. Ethylene-Induced Stabilization of ETHYLENE INSENSITIVE3 and

526 EIN3-LIKE1 Is Mediated by Proteasomal Degradation of EIN3 Binding F-Box 1 and 2 That

527 Requires EIN2 in Arabidopsis. Plant Cell 22, 2384-2401, doi:10.1105/tpc.110.076588 (2010).

5284 Basler, M., Ho, B. T. \& Mekalanos, J. J. Tit-for-tat: type VI secretion system

529 counterattack during bacterial cell-cell interactions. Cell 152, 884-894,

530 doi:10.1016/j.cell.2013.01.042 (2013).

5315 Bednarek, P. et al. A Glucosinolate Metabolism Pathway in Living Plant Cells

532 Mediates Broad-Spectrum Antifungal Defense. Science 323, 101-106,

533 doi:10.1126/science.1163732 (2009).

5346 Bok, J. W. \& Keller, N. P. LaeA, a regulator of secondary metabolism in Aspergillus 535 spp. Eukaryot Cell 3, 527-535, doi:10.1128/ec.3.2.527-535.2004 (2004).

5367 Bok, J. W., Noordermeer, D., Kale, S. P. \& Keller, N. P. Secondary metabolic gene

537 cluster silencing in Aspergillus nidulans. Mol Microbiol 61, 1636-1645, 538 doi:10.1111/j.1365-2958.2006.05330.x (2006).

5398 Bonfante, P. \& Genre, A. Mechanisms underlying beneficial plant-fungus

540 interactions in mycorrhizal symbiosis. Nat Commun 1, 48, doi:10.1038/ncomms1046 (2010).

5419 Bouhired, S., Weber, M., Kempf-Sontag, A., Keller, N. P. \& Hoffmeister, D.

542 Accurate prediction of the Aspergillus nidulans terrequinone gene cluster boundaries using the

543 transcriptional regulator LaeA. Fungal Genetics and Biology 44, 1134-1145,

544 doi:10.1016/j.fgb.2006.12.010 (2007).

54510 Bulgarelli, D., Schlaeppi, K., Spaepen, S., Ver Loren van Themaat, E. \&

546 Schulze-Lefert, P. Structure and functions of the bacterial microbiota of plants. Annu Rev Plant

547 Biol 64, 807-838, doi:10.1146/annurev-arplant-050312-120106 (2013).

54811 Bustos, R. et al. A central regulatory system largely controls transcriptional 549 activation and repression responses to phosphate starvation in Arabidopsis. PLoS Genet 6, 550 e1001102, doi:10.1371/journal.pgen.1001102 (2010). 
55112 Cannon, P. F., Damm, U., Johnston, P. R. \& Weir, B. S. Colletotrichum - current

552 status and future directions. Stud Mycol 73, 181-213, doi:10.3114/sim0014 (2012).

55313 Castrillo, G. et al. Root microbiota drive direct integration of phosphate stress and

554 immunity. Nature 543, 513-+, doi:10.1038/nature21417 (2017).

55514 de Lamo, F. J. \& Takken, F. L. W. Biocontrol by Fusarium oxysporum Using

556 Endophyte-Mediated Resistance. Front Plant Sci 11, 37, doi:10.3389/fpls.2020.00037 (2020).

55715 Dean, R. et al. The Top 10 fungal pathogens in molecular plant pathology. Mol Plant

558 Pathol 13, 414-430, doi:10.1111/j.1364-3703.2011.00783.x (2012).

$55916 \quad$ Druzhinina, I. S. et al. Trichoderma: the genomics of opportunistic success. Nat Rev

560 Microbiol 9, 749-759, doi:10.1038/nrmicro2637 (2011).

56117 Duran, P. et al. Microbial Interkingdom Interactions in Roots Promote Arabidopsis

562 Survival. Cell 175, 973-983 e914, doi:10.1016/j.cell.2018.10.020 (2018).

$56318 \quad$ Fu, Z. Q. \& Dong, X. N. Systemic Acquired Resistance: Turning Local Infection into

564 Global Defense. Annual Review of Plant Biology, Vol 64 64, 839-863,

565 doi:10.1146/annurev-arplant-042811-105606 (2013).

56619 Galagan, J. E. \& Selker, E. U. RIP: the evolutionary cost of genome defense. Trends 567 Genet 20, 417-423, doi:10.1016/j.tig.2004.07.007 (2004).

56820 Gan, P. et al. Comparative genomic and transcriptomic analyses reveal the 569 hemibiotrophic stage shift of Colletotrichum fungi. New Phytol 197, 1236-1249, 570 doi:10.1111/nph.12085 (2013).

57121 Gan, P. et al. Genus-Wide Comparative Genome Analyses of Colletotrichum Species

572 Reveal Specific Gene Family Losses and Gains during Adaptation to Specific Infection

573 Lifestyles. Genome Biol Evol 8, 1467-1481, doi:10.1093/gbe/evw089 (2016).

57422 Gan, P. et al. Genome Sequence Resources for Four Phytopathogenic Fungi from the

575 Colletotrichum orbiculare Species Complex. Mol Plant Microbe Interact 32, 1088-1090, 576 doi:10.1094/MPMI-12-18-0352-A (2019).

57723 Gao, F. K., Dai, C. C. \& Liu, X. Z. Mechanisms of fungal endophytes in plant 578 protection against path 
ogens. Afr J Microbiol Res 4, 1346-1351 (2010).

58124 García, E., Alonso, Á., Platas, G. \& Sacristán, S. The endophytic mycobiota of

582 Arabidopsis thaliana. Fungal Diversity 60, 71-89, doi:10.1007/s13225-012-0219-0 (2012).

58325 Gulshan, K. \& Moye-Rowley, W. S. Multidrug resistance in fungi. Eukaryot Cell 6, 584 1933-1942, doi:10.1128/Ec.00254-07 (2007).

$58526 \quad$ Hacquard, S. et al. Survival trade-offs in plant roots during colonization by closely

586 related beneficial and pathogenic fungi. Nature Communications 7, doi:ARTN 11362

587 10.1038/ncomms11362 (2016).

$58827 \quad$ Hiruma, K. et al. Root Endophyte Colletotrichum tofieldiae Confers Plant Fitness

589 Benefits that Are Phosphate Status Dependent. Cell 165, 464-474,

590 doi:10.1016/j.cell.2016.02.028 (2016).

59128 Hiruma, K., Kobae, Y. \& Toju, H. Beneficial associations between Brassicaceae 592 plants and fungal endophytes under nutrient-limiting conditions: evolutionary origins and 593 host-symbiont molecular mechanisms. Curr Opin Plant Biol 44, 145-154, 594 doi:10.1016/j.pbi.2018.04.009 (2018).

$59529 \quad$ Jacoby, R., Peukert, M., Succurro, A., Koprivova, A. \& Kopriva, S. The Role of Soil

596 Microorganisms in Plant Mineral Nutrition-Current Knowledge and Future Directions. Front 597 Plant Sci 8, 1617, doi:10.3389/fpls.2017.01617 (2017).

$59830 \quad$ Kale, S. P. et al. Requirement of LaeA for secondary metabolism and sclerotial 599 production in Aspergillus flavus. Fungal Genetics and Biology 45, 1422-1429, 600 doi:10.1016/j.fgb.2008.06.009 (2008).

$60131 \quad$ Kavroulakis, N. et al. Role of ethylene in the protection of tomato plants against 602 soil-borne fungal pathogens conferred by an endophytic Fusarium solani strain. J Exp Bot 58, 603 3853-3864, doi:10.1093/jxb/erm230 (2007).

60432 Kim, D., Landmead, B. \& Salzberg, S. L. HISAT: a fast spliced aligner with low 605 memory requirements. Nat Methods 12, 357-U121, doi:10.1038/Nmeth.3317 (2015).

60633 Kim, W. J. et al. Commensal Neisseria Kill Neisseria gonorrhoeae through a 607 DNA-Dependent Mechanism. Cell Host Microbe 26, 228-+, doi:10.1016/j.chom.2019.07.003 608 (2019).

60934 Kloppholz, S., Kuhn, H. \& Requena, N. A secreted fungal effector of Glomus 610 intraradices promotes symbiotic biotrophy. Curr Biol 21, 1204-1209, 611 doi:10.1016/j.cub.2011.06.044 (2011). 
61235 Konig, J., Muller, F. \& Fromm, M. F. Transporters and drug-drug interactions:

613 important determinants of drug disposition and effects. Pharmacol Rev 65, 944-966,

614 doi:10.1124/pr.113.007518 (2013).

$61536 \quad$ Kunzler, M. How fungi defend themselves against microbial competitors and animal

616 predators. Plos Pathog 14, doi:ARTN e100718410.1371/journal.ppat.1007184 (2018).

$61737 \quad$ Lipka, V. et al. Pre- and postinvasion defenses both contribute to nonhost resistance 618 in Arabidopsis. Science 310, 1180-1183, doi:10.1126/science.1119409 (2005).

61938 Liu, Y. et al. Light and Ethylene Coordinately Regulate the Phosphate Starvation

620 Response through Transcriptional Regulation of PHOSPHATE STARVATION RESPONSE1.

621 Plant Cell 29, 2269-2284, doi:10.1105/tpc.17.00268 (2017).

62239 Lo Presti, L. et al. Fungal effectors and plant susceptibility. Annu Rev Plant Biol 66, 623 513-545, doi:10.1146/annurev-arplant-043014-114623 (2015).

$62440 \quad$ Lodeiro, S. et al. Protostadienol Biosynthesis and Metabolism in the Pathogenic

625 Fungus Aspergillus fumigatus. Org Lett 11, 1241-1244, doi:10.1021/o1802696a (2009).

62641 Lugtenberg, B. \& Kamilova, F. Plant-growth-promoting rhizobacteria. Annu Rev

627 Microbiol 63, 541-556, doi:10.1146/annurev.micro.62.081307.162918 (2009).

62842 Lundberg, D. S. et al. Defining the core Arabidopsis thaliana root microbiome. 629 Nature 488, 86-90, doi:10.1038/nature11237 (2012).

63043 Ma, L. S., Hachani, A., Lin, J. S., Filloux, A. \& Lai, E. M. Agrobacterium 631 tumefaciens deploys a superfamily of type VI secretion DNase effectors as weapons for 632 interbacterial competition in planta. Cell Host Microbe 16, 94-104, 633 doi:10.1016/j.chom.2014.06.002 (2014).

63444 Macheleidt, J. et al. Regulation and Role of Fungal Secondary Metabolites. Annu 635 Rev Genet 50, 371-392, doi:10.1146/annurev-genet-120215-035203 (2016).

63645 Millet, Y. A. et al. Innate Immune Responses Activated in Arabidopsis Roots by 637 Microbe-Associated Molecular Patterns. Plant Cell 22, 973-990, doi:10.1105/tpc.109.069658 638 (2010).

63946 Moran-Diez, E. et al. Transcriptomic response of Arabidopsis thaliana after $24 \mathrm{~h}$ 640 incubation with the biocontrol fungus Trichoderma harzianum. J Plant Physiol 169, 614-620, 641 doi:10.1016/j.jplph.2011.12.016 (2012).

64247 Morrissey, J. P. \& Osbourn, A. E. Fungal resistance to plant antibiotics as a 643 mechanism of pathogenesis. Microbiol Mol Biol R 63, 708-+, doi:Doi $644 \quad$ 10.1128/Mmbr.63.3.708-724.1999 (1999). 
$64548 \quad$ Nishi, T. \& Itoh, S. Enhancement of Transcriptional Activity of the Escherichia-Coli

646 Trp Promoter by Upstream A+T-Rich Regions. Gene 44, 29-36, doi:Doi

647 10.1016/0378-1119(86)90039-9 (1986).

64849 O'Connell, R. et al. A novel Arabidopsis-Colletotrichum pathosystem for the

649 molecular dissection of plant-fungal interactions. Mol Plant Microbe In 17, 272-282, doi:Doi

650 10.1094/Mpmi.2004.17.3.272 (2004).

$65150 \quad$ O'Connell, R. J. et al. Lifestyle transitions in plant pathogenic Colletotrichum fungi

652 deciphered by genome and transcriptome analyses. Nat Genet 44, 1060-1065, 653 doi:10.1038/ng.2372(2012).

$65451 \quad$ Palida, F., Hale, C., and Sprague, K.V. Transcription of a silk- worm tRNA(cAla) is 655 directed by two AT-rich upstream sequence elements. Nucleic Acids Res. 21, 5875-5881 656 (1993).

65752 Palmer, J. M. et al. Secondary Metabolism and Development Is Mediated by LlmF 658 Control of VeA Subcellular Localization in Aspergillus nidulans. Plos Genetics 9, doi:ARTN 659 e1003193

660 10.1371/journal.pgen.1003193 (2013).

$66153 \quad$ Perfect, S. E., Hughes, H. B., O'Connell, R. J. \& Green, J. R. Colletotrichum: A 662 model genus for studies on pathology and fungal-plant interactions. Fungal Genet Biol 27, 663 186-198, doi:10.1006/fgbi.1999.1143 (1999).

66454 Pickard, J. M., Zeng, M. Y., Caruso, R. \& Nunez, G. Gut microbiota: Role in 665 pathogen colonization, immune responses, and inflammatory disease. Immunol Rev 279, 70-89, 666 doi:10.1111/imr.12567 (2017).

$66755 \quad$ Pieterse, C. M., Van der Does, D., Zamioudis, C., Leon-Reyes, A. \& Van Wees, S. C. 668 Hormonal modulation of plant immunity. Annu Rev Cell Dev Biol 28, 489-521, 669 doi:10.1146/annurev-cellbio-092910-154055 (2012).

$67056 \quad$ Pieterse, C. M. J. et al. Induced Systemic Resistance by Beneficial Microbes. Annual 671 Review of Phytopathology, Vol 52 52, 347-375, doi:10.1146/annurev-phyto-082712-102340 672 (2014).

67357 Prasad, R. \& Goffeau, A. Yeast ATP-binding cassette transporters conferring 674 multidrug resistance. Annu Rev Microbiol 66, 39-63, 675 doi:10.1146/annurev-micro-092611-150111 (2012).

67658 Raffaele, S. \& Kamoun, S. Genome evolution in filamentous plant pathogens: why 677 bigger can be better. Nat Rev Microbiol 10, 417-430, doi:10.1038/nrmicro2790 (2012). 
67859 Reyes-Dominguez, Y. et al. Heterochromatic marks are associated with the

679 repression of secondary metabolism clusters in Aspergillus nidulans. Mol Microbiol 76, 680 1376-1386, doi:10.1111/j.1365-2958.2010.07051.x (2010).

$68160 \quad$ Robert-Seilaniantz, A., Grant, M. \& Jones, J. D. Hormone crosstalk in plant disease

682 and defense: more than just jasmonate-salicylate antagonism. Annu Rev Phytopathol 49, 683 317-343, doi:10.1146/annurev-phyto-073009-114447 (2011).

$68461 \quad$ Rodriguez, R. J., White, J. F., Jr., Arnold, A. E. \& Redman, R. S. Fungal endophytes: 685 diversity and functional roles. New Phytol 182, 314-330, 686 doi:10.1111/j.1469-8137.2009.02773.x (2009).

68762 Rubio, V. et al. A conserved MYB transcription factor involved in phosphate 688 starvation signaling both in vascular plants and in unicellular algae. Gene Dev 15, 2122-2133, 689 doi:DOI 10.1101/gad.204401 (2001).

69063 Rouxel, T. et al. Effector diversification within compartments of the Leptosphaeria 691 maculans genome affected by Repeat-Induced Point mutations. Nature Communications 2, 692 doi:10.1038/ncomms1189 (2011).

69364 Ruocco, M. et al. Identification of a New Biocontrol Gene in Trichoderma 694 atroviride: The Role of an ABC Transporter Membrane Pump in the Interaction with Different 695 Plant-Pathogenic Fungi. Mol Plant Microbe In 22, 291-301, doi:10.1094/Mpmi-22-3-0291 696 (2009).

69765 Sanchez-Vallet, A. et al. The Genome Biology of Effector Gene Evolution in 698 Filamentous Plant Pathogens. Annu Rev Phytopathol 56, 21-40, 699 doi:10.1146/annurev-phyto-080516-035303 (2018).

$70066 \quad$ Sarkar, D. et al. The inconspicuous gatekeeper: endophytic Serendipita vermifera 701 acts as extended plant protection barrier in the rhizosphere. New Phytol 224, 886-901, 702 doi:10.1111/nph.15904 (2019).

70367 Sato, T. et al. Anthracnose of Japanese radish caused by Colletotrichum dematium. J

704 Gen Plant Pathol 71, 380-383, doi:10.1007/s10327-005-0214-3 (2005).

70568 Schardl, C. L. et al. Plant-symbiotic fungi as chemical engineers: multi-genome 706 analysis of the clavicipitaceae reveals dynamics of alkaloid loci. PLoS Genet 9, e1003323, 707 doi:10.1371/journal.pgen.1003323 (2013).

70869 Schoch, C. L. et al. Nuclear ribosomal internal transcribed spacer (ITS) region as a 709 universal DNA barcode marker for Fungi. P Natl Acad Sci USA 109, 6241-6246, 710 doi:10.1073/pnas.1117018109 (2012). 
$71170 \quad$ Schuster, A. \& Schmoll, M. Biology and biotechnology of Trichoderma. Appl

712 Microbiol Biot 87, 787-799, doi:10.1007/s00253-010-2632-1 (2010).

71371 Song, L. et al. The Molecular Mechanism of Ethylene-Mediated Root Hair

714 Development Induced by Phosphate Starvation. PLoS Genet 12, e1006194,

715 doi:10.1371/journal.pgen.1006194 (2016).

71672 Souza, D. P. et al. Bacterial killing via a type IV secretion system. Nat Commun 6, 717 6453, doi:10.1038/ncomms7453 (2015).

$71873 \quad$ Stein, E., Molitor, A., Kogel, K. H. \& Waller, F. Systemic resistance in Arabidopsis

719 conferred by the mycorrhizal fungus Piriformospora indica requires jasmonic acid signaling

720 and the cytoplasmic function of NPR1. Plant Cell Physiol 49, 1747-1751, 721 doi:10.1093/pcp/pcn147 (2008).

$72274 \quad$ Stroe, M. C. et al. Targeted induction of a silent fungal gene cluster encoding the 723 bacteria-specific germination inhibitor fumigermin. Elife 9, doi:10.7554/eLife.52541 (2020).

72475 Thomma, B. P. H. J., Penninckx, I. A. M. A., Broekaert, W. F. \& Cammue, B. P. A.

725 The complexity of disease signaling in Arabidopsis. Curr Opin Immunol 13, 63-68, doi:Doi

726 10.1016/S0952-7915(00)00183-7 (2001).

72776 Toju, H. et al. Core microbiomes for sustainable agroecosystems. Nat Plants 4, 728 247-257, doi:10.1038/s41477-018-0139-4 (2018).

72977 Trunk, K. et al. The type VI secretion system deploys antifungal effectors against 730 microbial competitors. Nat Microbiol 3, 920-931, doi:10.1038/s41564-018-0191-x (2018).

73178 Van Wees, S. C., Van der Ent, S. \& Pieterse, C. M. Plant immune responses 732 triggered by beneficial microbes. Curr Opin Plant Biol 11, 443-448, 733 doi:10.1016/j.pbi.2008.05.005 (2008).

73479 Walker, L. A., Gow, N. A. R. \& Munro, C. A. Fungal echinocandin resistance. 735 Fungal Genetics and Biology 47, 117-126, doi:10.1016/j.fgb.2009.09.003 (2010).

$73680 \quad$ Wang, H. et al. Horizontal gene transfer of Fhb7 from fungus underlies Fusarium 737 head blight resistance in wheat. Science 368, doi:10.1126/science.aba5435 (2020).

$73881 \quad$ Weir, B. S., Johnston, P. R. \& Damm, U. The Colletotrichum gloeosporioides 739 species complex. Stud Mycol 73, 115-180, doi:10.3114/sim0011 (2012).

74082 Wiemann, P. et al. Biosynthesis of the red pigment bikaverin in Fusarium fujikuroi: 741 genes, their function and regulation. Molecular Microbiology 72, 931-946, 742 doi:10.1111/j.1365-2958.2009.06695.x (2009). 
$74383 \quad \mathrm{Xu}, \mathrm{X} . \mathrm{H}$. et al. The rice endophyte Harpophora oryzae genome reveals evolution

744 from a pathogen to a mutualistic endophyte. Sci Rep 4, 5783, doi:10.1038/srep05783 (2014).

$74584 \quad$ Zamioudis, C. \& Pieterse, C. M. Modulation of host immunity by beneficial

746 microbes. Mol Plant Microbe Interact 25, 139-150, doi:10.1094/MPMI-06-11-0179 (2012).

$74785 \quad$ Zhang, L. Q. et al. Novel Fungal Pathogenicity and Leaf Defense Strategies Are

748 Revealed by Simultaneous Transcriptome Analysis of Colletotrichum fructicola and Strawberry

749 Infected by This Fungus. Frontiers in Plant Science 9, doi:ARTN 434

$750 \quad 10.3389 /$ fpls.2018.00434 (2018).

$75186 \quad$ Zhang, Q. H. et al. Diversity and biocontrol potential of endophytic fungi in Brassica

752 napus. Biol Control 72, 98-108, doi:10.1016/j.biocontrol.2014.02.018 (2014).

75387 Zhao, Y. D. et al. Trp-dependent auxin biosynthesis in Arabidopsis: involvement of

754 cytochrome P450s CYP79B2 and CYP79B3. Gene Dev 16, 3100-3112, 755 doi:10.1101/gad.1035402 (2002).

$75688 \quad$ Zhou, N., Tootle, T. L. \& Glazebrook, J. Arabidopsis PAD3, a gene required for 757 camalexin biosynthesis, encodes a putative cytochrome P450 monooxygenase. Plant Cell 11, 758 2419-2428, doi:DOI 10.1105/tpc.11.12.2419(1999).

$75989 \quad$ Zivkovic, S. et al. Screening of Antagonistic Activity of Microorganisms against 760 Colletotrichum Acutatum and Colletotrichum Gloeosporioides. Arch Biol Sci 62, 611-623, 761 doi:10.2298/Abs1003611z (2010).

762

Fig legends

764

Fig. 1. Endophytic CgE protects Arabidopsis thaliana plants from the closely-related 766 pathogenic CgP

767 (A) Representative pictures of plants treated with water, endophytic Colletotrichum (CgE), and pathogenic Colletotrichum $(\mathrm{CgP})$ or co-inoculated with $\mathrm{CgE}$ and $\mathrm{CgP}$ at $21 \mathrm{dpi}$ on 1/2 MS agar media. (B) Shoot fresh weight of Arabidopsis thaliana from the co-inoculation assay at $21 \mathrm{dpi}$. Each sample comprised at least 10 shoots per experiment. The boxplot shows combined data

771 from two independent experiments. The dots indicate individual replicates. Different letters 772 indicate significantly different statistical groups (Tukey's HSD, p $<0.05$ ). (C) Phylogenetic 773 positions of $\mathrm{CgE}$ and $\mathrm{CgP}$. Phylogenetic tree of the concatenated protein-coding gene sequences 774 for 16 Colletotrichum species. Multiple alignments of 4,650 ortholog groups were concatenated, 775 and a phylogenetic tree was constructed by using 94,749 amino acid sites. Bootstrap values are 
776 shown on the branches. $\mathrm{CgE}$ and $\mathrm{CgP}$ belong to gloeosporioides clade. (D) One of the AT rich

777 regions in $\mathrm{CgE}$ genome. AT block gene corresponds to CGE00232 gene. The genomic

778 sequences surrounding the CGE00232 gene were extracted from the genome assembly of each

779 allele in $\mathrm{CgE}$ and $\mathrm{CgP}$. Vertical bars connecting adjacent genomic structures indicate BLAST

780 hit blocks in the comparison between the two adjacent genomic scaffolds. Orange polygons

781 indicate predicted genes. Red arrow indicates CGE00232 gene. Sequences of CGE00232 gene

782 (Red arrow) and the dashed square regions show high similarity to CGP01804 (Black arrow).

783 RIP indexes of $\mathrm{CgP}$ (upper) and $\mathrm{CgE}$ (lower) are also described. RIP index values depicted: RIP

784 product (green), RIP substrate (yellow) and RIP composite (red). RIP composite index values

785 exceeding 0 indicate RIP activity. GC rate indicates GC content per $1 \mathrm{~kb}$ window. (E) Numbers of shared and specific orthologous family genes in $\mathrm{CgE}$ and $\mathrm{CgP}$.

787

788

789

Fig. 2. Endophytic CgE inhibits the growth of the pathogen CgP on Arabidopsis thaliana roots

(A) Colony morphology of $\mathrm{CgE}$ and $\mathrm{CgP}$ at 7 dpi on PDA medium plates. Fungal colonies were placed beside another fungus, a PDA plug, and filter paper disc containing water or Hygormycin B. In contrast to Hygormycin B treatment, which formed an inhibition zone in front of $\mathrm{CgP}$ colony, $\mathrm{CgE}$ did not induce an inhibition zone in front of $\mathrm{CgP}$.

(B) A fungal biomass for $\mathrm{CgE}$ or $\mathrm{CgP}$ in roots was determined by $\mathrm{qPCR}$ using primers that specifically detect $\mathrm{CgE}$ or $\mathrm{CgP}$ genomes, respectively at $3 \mathrm{dpi}$. Bars represent means and standard deviation (SD) of data collected in 4 different root mixed samples (each sample comprised $20-25$ roots) ( ${ }^{*}$-test, $p<0.01$ ). (C) Confocal microscope images of $\mathrm{CgP}$ expressing cytoplasmic GFP (green) and Arabidopsis thaliana stained with propidium iodide (red). The hyphal network of CgP-GFP colonized around the root (left panel) and co-colonized with CgE-wild-type (Right panel). Arrows indicate the newly generated spores of CgP-GFP in the Arabidopsis thaliana root at 3 dpi. Scale bar, $100 \mu \mathrm{m}$.

(D) Confocal microscope images of $\mathrm{CgP}$ and Arabidopsis thaliana plants. CgP formed spores (Left, dashed arrow) via black melanized structures (Left, black arrow). However, $\mathrm{CgE}$ inoculation inhibited formation of black melanized structures.

Fig. 3. Endophytic CgE colonization and host-protective function are phosphate status dependent 
809 fresh weight of Arabidopsis thaliana wild-type plants (Col-0) from the co-inoculation assay at

81021 dpi. Similar results have been obtained in independent experiments. Different letters indicate

811 significantly different statistical groups (Tukey's HSD, $\mathrm{p}<0.05$ ). M= Mock. (C) Morphology

812 of plants treated with water, $\mathrm{CgE}, \mathrm{CgP}$ or co-inoculation of $\mathrm{CgE}$ with $\mathrm{CgP}$ on $1 / 2 \mathrm{MS}$ Low Pi

$813(50 \mu \mathrm{M})$ agar media.

815 Fig. 4. Endophytic CgE colonization and host protection require plant ethylene signaling

817 (A) Morphology of the plants treated with water, $\mathrm{CgE}, \mathrm{CgP}$ or co-inoculation of $\mathrm{CgE}$ with $\mathrm{CgP}$ $818(\mathrm{CgE}+\mathrm{CgP})$ at $21 \mathrm{dpi}$ on $1 / 2 \mathrm{MS}$ agar media. (B) Measurement of the shoot fresh weight of 819 wild-type Arabidopsis thaliana and ET-related mutants (ein2-1, ein3) in the co-inoculation 820 assay at 14 dpi. Each sample comprised around 20 shoots per experiment. The boxplot shows 821 combined data from three independent experiments. Different letters indicate significantly different statistical groups (Tukey's HSD, $p<0.05$ ).

823

824 Fig. 5. Endophytic CgE colonization and host protection require host tryptophan 825 (Trp)-derived metabolites

826 (A) Scheme for Trp-derived metabolite pathways in Arabidopsis thaliana. (B) Measurement 827 of the shoot fresh weight of Arabidopsis thaliana Trp-pathway mutant plants treated with water, $828 \mathrm{CgE}$ and $\mathrm{CgP}$ or co-inoculated with $\mathrm{CgE}$ and $\mathrm{CgP}$ at 14 dpi. The boxplot shows combined data 829 from three independent experiments. Different letters indicate significantly different statistical 830 groups (Tukey's HSD, $p<0.05$ ).

832 Fig. 6. Transcriptome analysis of fungi detects large gene expression changes in 833 co-inoculated samples

834 (A) Schematic diagram of the classification of RNA-seq reads from co-inoculated samples. The 835 table represents a number of $\mathrm{CgE}$ or $\mathrm{CgP}$ genes specifically upregulated or downregulated in co-inoculated samples compared with that in the corresponding single-inoculated samples at 3

837 dpi. DEGs = differentially expressed genes. (B) Transcript profiling of $74 \mathrm{CgE}$ SSP DEGs $838\left(\left|\log _{2} \mathrm{FC}\right| \geqslant 1, \quad \mathrm{FDR}<0.05\right)$ between $\mathrm{CgE}$-colonized versus (vs) $\mathrm{CgE}+\mathrm{CgP}$-colonized roots. 839 Overrepresented (yellow to red) and underrepresented transcripts (yellow to blue) are shown as $840 \log _{10}$ (read count +1$)$. LogFC_CgP represents $\log \mathrm{FC}(\mathrm{CgP}-$ colonized vs $\mathrm{CgE}+\mathrm{CgP}$-colonized 841 roots) of the corresponding $\mathrm{CgP}$ genes (Blue to Red). White represents the absence of obvious 
842 homologs in CgP (Similarity $<90 \%)$. FDR_CgP represents whether the expression levels of

843 the corresponding $\mathrm{CgP}$ genes between $\mathrm{CgP}$-colonized and $\mathrm{CgE}+\mathrm{CgP}$-colonized roots are

844 significant (Blue: FDR $<0.05$, Black: FDR $>0.05$, White: no homologs in $\mathrm{CgP}$ (Similarity $<$

845 90\%). (C) Results of Gene ontology (GO) analysis using $721 \mathrm{CgE}$ up-regulated DEGs in the

846 co-inoculated samples. The enriched GO terms of biological process were shown. (D) The

847 expression profiles of $\mathrm{CgE}$ genes located in the secondary metabolism 25 cluster. The genomic

848 sequences surrounding the secondary metabolism 25 cluster were extracted from the genome

849 assembly of each allele in $\mathrm{CgE}$ and $\mathrm{CgP}$. Vertical bars connecting adjacent genomic structures

850 indicate BLAST hit blocks in the comparison between the two adjacent genomic scaffolds.

851 Polygons indicate predicted genes. The red represents significantly higher expression in the

852 co-inoculated samples compared to its alone $\left(\log _{2} \mathrm{FC}>1\right.$, FDR $\left.<0.05\right)$. GC rate indicates GC

853 content per $1 \mathrm{~kb}$ window. 



Mock

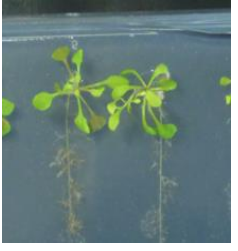

$\mathrm{CgE}$

$\mathrm{CgP}$

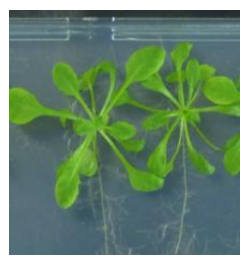

$\mathrm{CgE}+\mathrm{CgP}$

C

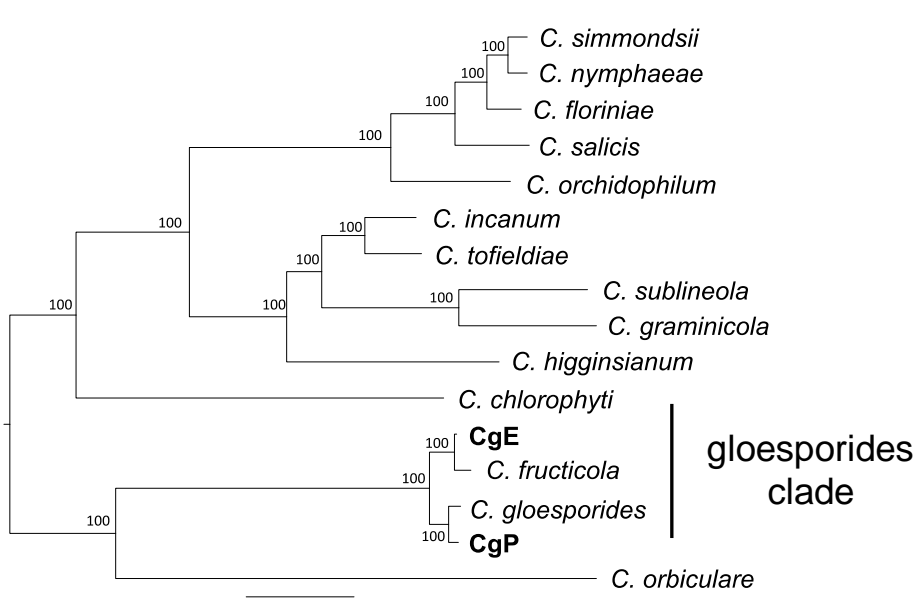

D

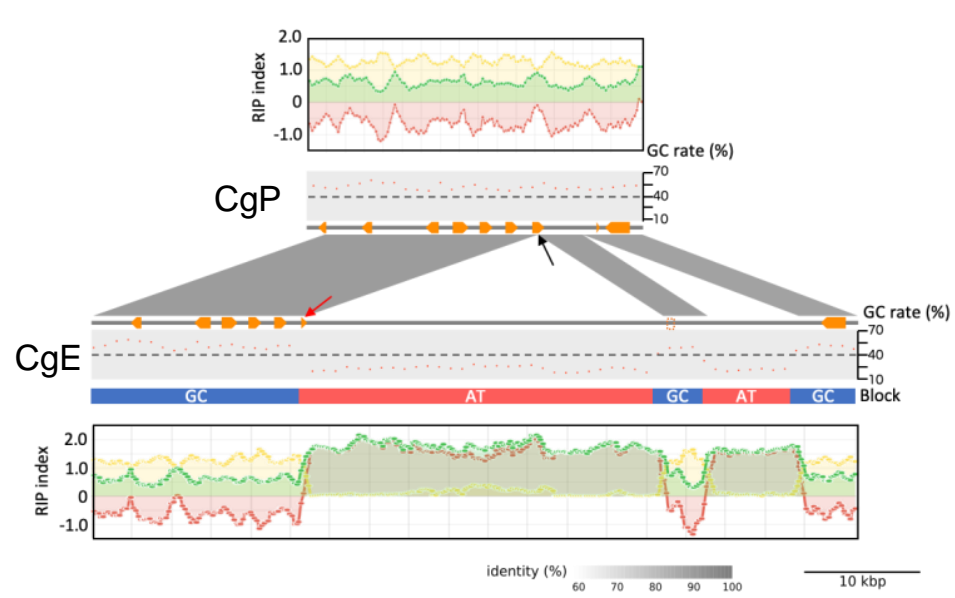

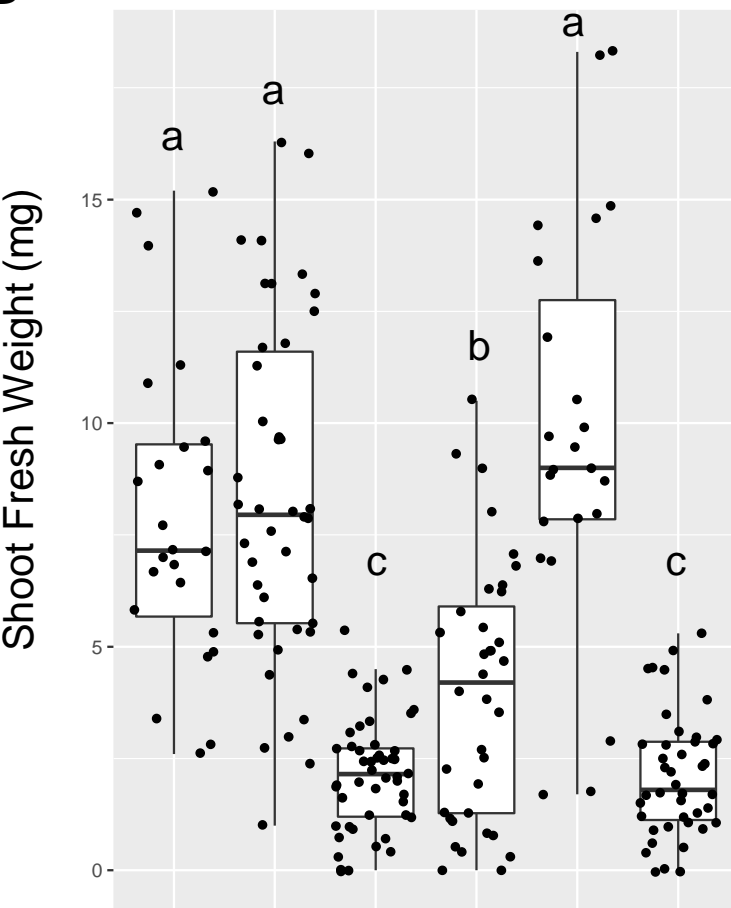

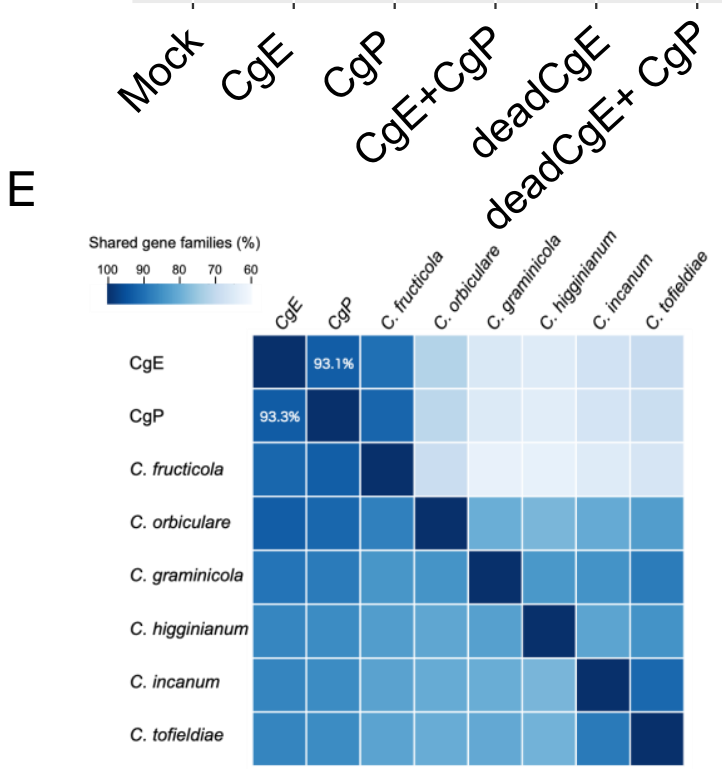

Fig. 1. Endophytic $\mathrm{CgE}$ protects Arabidopsis thaliana plants from the closely-related pathogenic $\mathrm{CgP}$ (A) Representative pictures of plants treated with water, endophytic Colletotrichum ( $\mathrm{CgE}$ ), and pathogenic Colletotrichum (CgP) or co-inoculated with $\mathrm{CgE}$ and $\mathrm{CgP}$ at 21 dpi on 1/2 MS agar media. (B) Shoot fresh weight of Arabidopsis from the co-inoculation assay at $21 \mathrm{dpi}$. Each sample comprised at least 10 shoots per experiment. The boxplot shows combined data from two independent experiments. The dots indicate individual replicates. Different letters indicate significantly different statistical groups (Tukey's HSD, $p<0.05$ ). (C) Phylogenetic positions of $\mathrm{CgE}$ and $\mathrm{CgP}$. Phylogenetic tree of the concatenated protein-coding gene sequences for 16 Colletotrichum species. Multiple alignments of 4,650 ortholog groups were concatenated, and a phylogenetic tree was constructed by using 94,749 amino acid sites. Bootstrap values are shown on the branches. $\mathrm{CgE}$ and $\mathrm{CgP}$ belong to gloeosporioides clade. (D) One of the AT rich regions in $\mathrm{CgE}$ genome. AT block gene corresponds to CGE00232 gene. The genomic sequences surrounding the CGE00232 gene were extracted from the genome assembly of each allele in $\mathrm{CgE}$ and $\mathrm{CgP}$. Vertical bars connecting adjacent genomic structures indicate BLAST hit blocks in the comparison between the two adjacent genomic scaffolds. Orange polygons indicate predicted genes. Red arrow indicates CGE00232 gene. Sequences of CGE00232 gene (Red arrow) and the dashed square regions show high similarity to CGP01804 (Black arrow). RIP indexes of $\mathrm{CgP}$ (upper) and $\mathrm{CgE}$ (lower) are also described. RIP index values depicted: RIP product (green), RIP substrate (yellow) and RIP composite (red). RIP composite index values exceeding 0 are indicate RIP activity. GC rate indicates GC content per $1 \mathrm{~kb}$ window. (E) Numbers of shared and specific orthologous family genes in $\mathrm{CgE}$ and $\mathrm{CgP}$. 
A


B
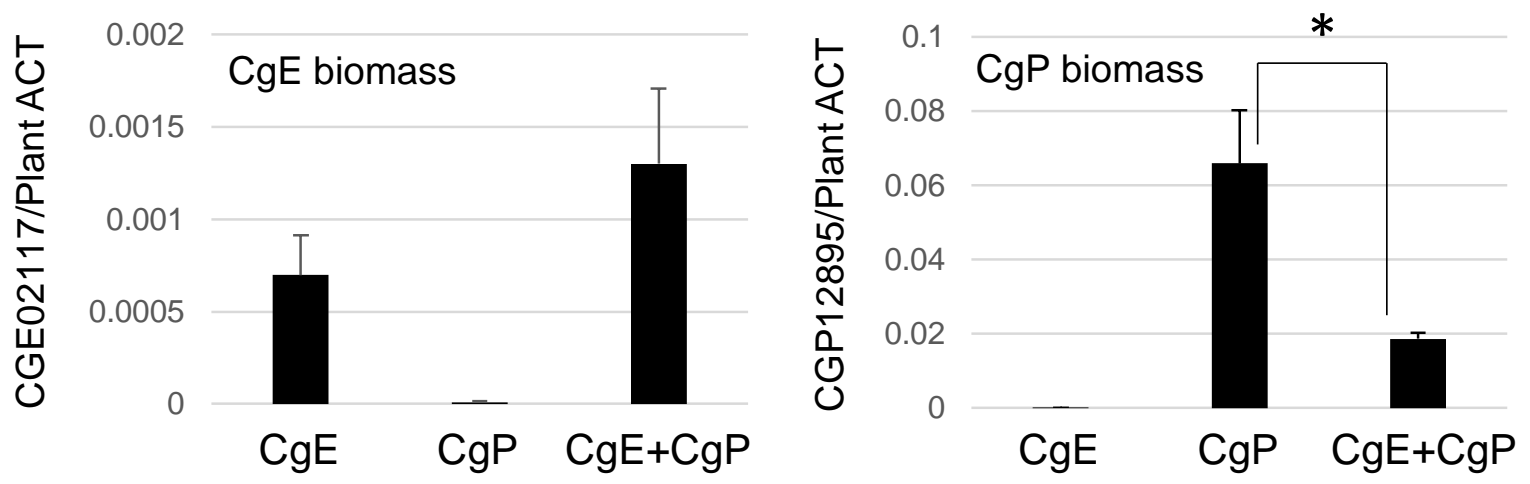

C


D

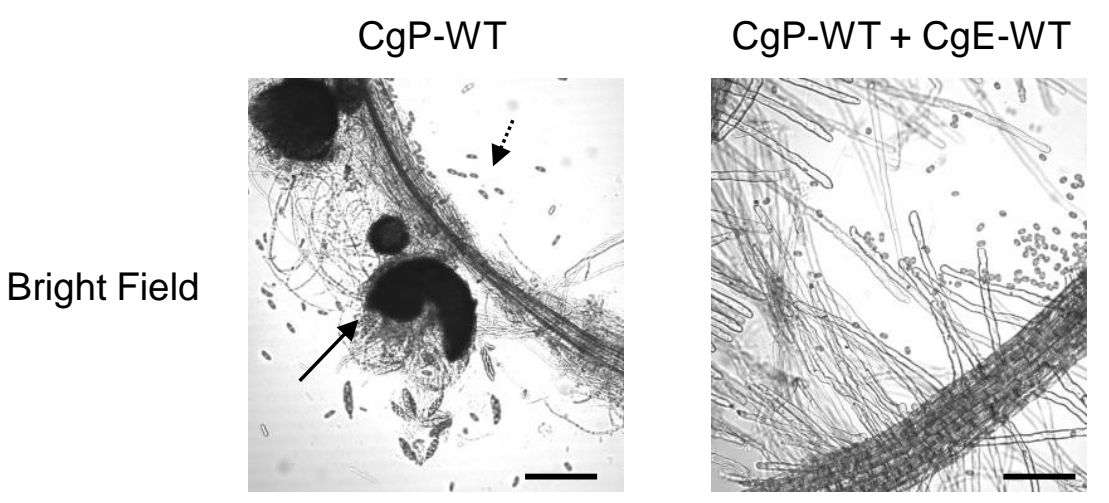

Fig. 2. Endophytic $\mathrm{CgE}$ inhibits the growth of the pathogen $\mathrm{CgP}$ on Arabidopsis thaliana roots (A) Colony morphology of $\mathrm{CgE}$ and $\mathrm{CgP}$ at $7 \mathrm{dpi}$ on PDA medium plates. Fungal colonies were placed beside another fungus, a PDA plug, and filter paper disc containing water or Hygormycin B. In contrast to Hygormycin B treatment, which formed an inhibition zone in front of $\mathrm{CgP}$ colony, $\mathrm{CgE}$ did not induce an inhibition zone in front of $\mathrm{CgP}$. (B) A fungal biomass for $\mathrm{CgE}$ or $\mathrm{CgP}$ in roots was determined by qPCR using primers that specifically detect $\mathrm{CgE}$ or $\mathrm{CgP}$ genomes, respectively at 3 dpi. Bars represent means and standard deviation (SD) of data collected in 4 different root mixed samples (each sample comprised $20-25$ roots) ( ${ }^{*} t$-test, $\left.p<0.01\right)$. (C) Confocal microscope images of $\mathrm{CgP}$ expressing cytoplasmic GFP (green) and $A$. thaliana stained with propidium iodide (red). The hyphal network of CgP-GFP colonized around the root (left panel) and co-colonized with CgE-wildtype (Right panel). Arrows indicate the newly generated spores of CgP-GFP in the $A$. thaliana root at 3 dpi. Scale bar, $100 \mu \mathrm{m}$. (D) Confocal microscope images of $\mathrm{CgP}$ and $A$. thaliana plants. $\mathrm{CgP}$ formed spores (Left, dashed arrow) via black melanized structures (Left, black arrow). However, $\mathrm{CgE}$ inoculation inhibited formation of black melanized structures. 

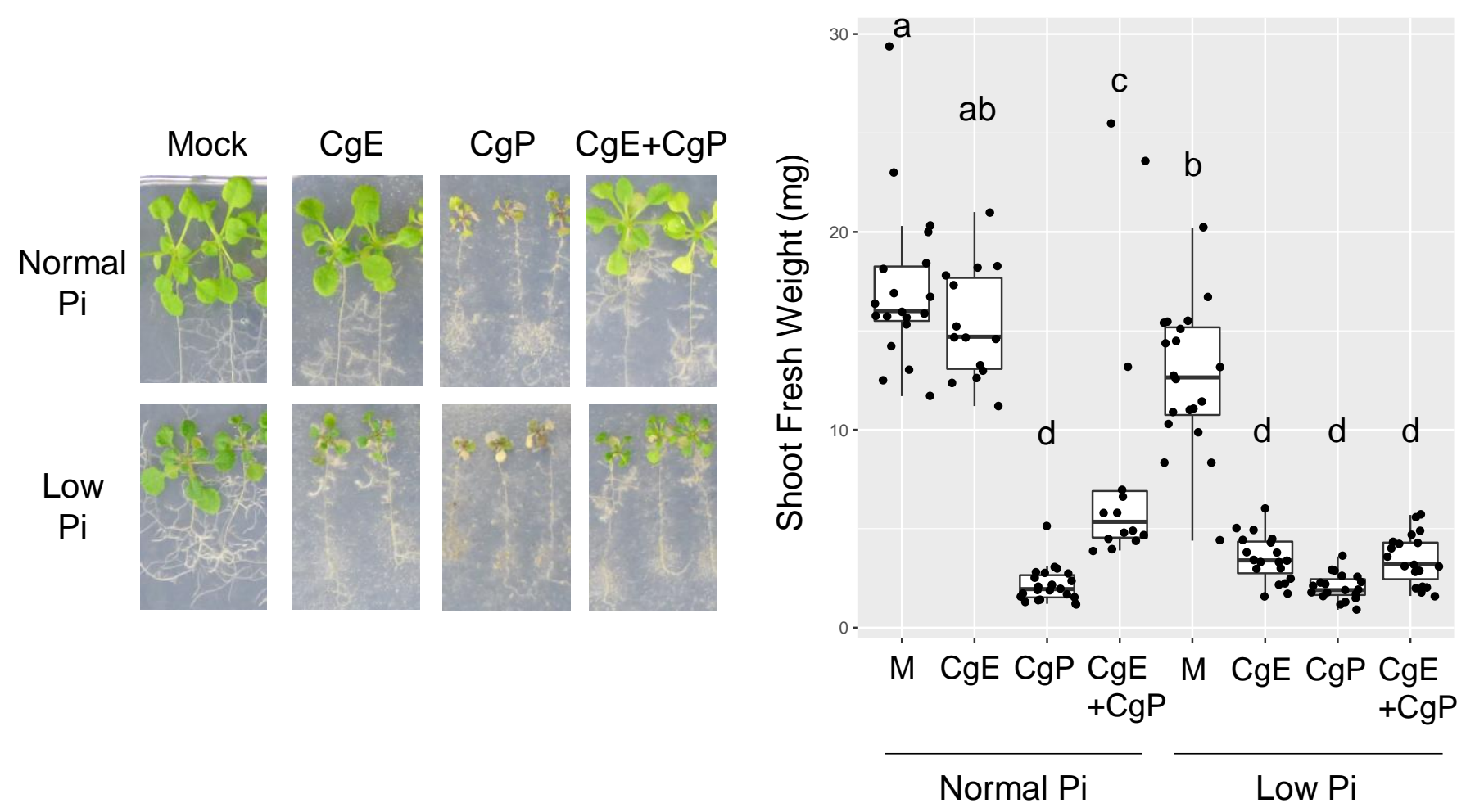

C



Fig. 3. Endophytic $\mathrm{CgE}$ colonization and host-protective function are phosphate status dependent

(A) Morphology of plants treated with water, $\mathrm{CgE}, \mathrm{CgP}$ or co-inoculation of $\mathrm{CgE}$ with $\mathrm{CgP}$ $(\mathrm{CgE}+\mathrm{CgP})$ at $21 \mathrm{dpi}$ on 1/2 MS agar normal $\mathrm{Pi}(625 \mu \mathrm{M})$ and low $\mathrm{Pi}(50 \mu \mathrm{M})$ media. (B) Shoot fresh weight of Arabidopsis wild-type plants (Col-0) from the co-inoculation assay at $21 \mathrm{dpi}$. Similar results have been obtained in independent experiments. Different letters indicate significantly different statistical groups (Tukey's HSD, $\mathrm{p}<0.05$ ). $M=$ Mock. (C) Morphology of plants treated with water, $\mathrm{CgE}, \mathrm{CgP}$ or co-inoculation of $\mathrm{CgP}$ with $\mathrm{CgE}$ on 1/2 MS Low Pi $(50 \mu \mathrm{M})$ agar media. 
A

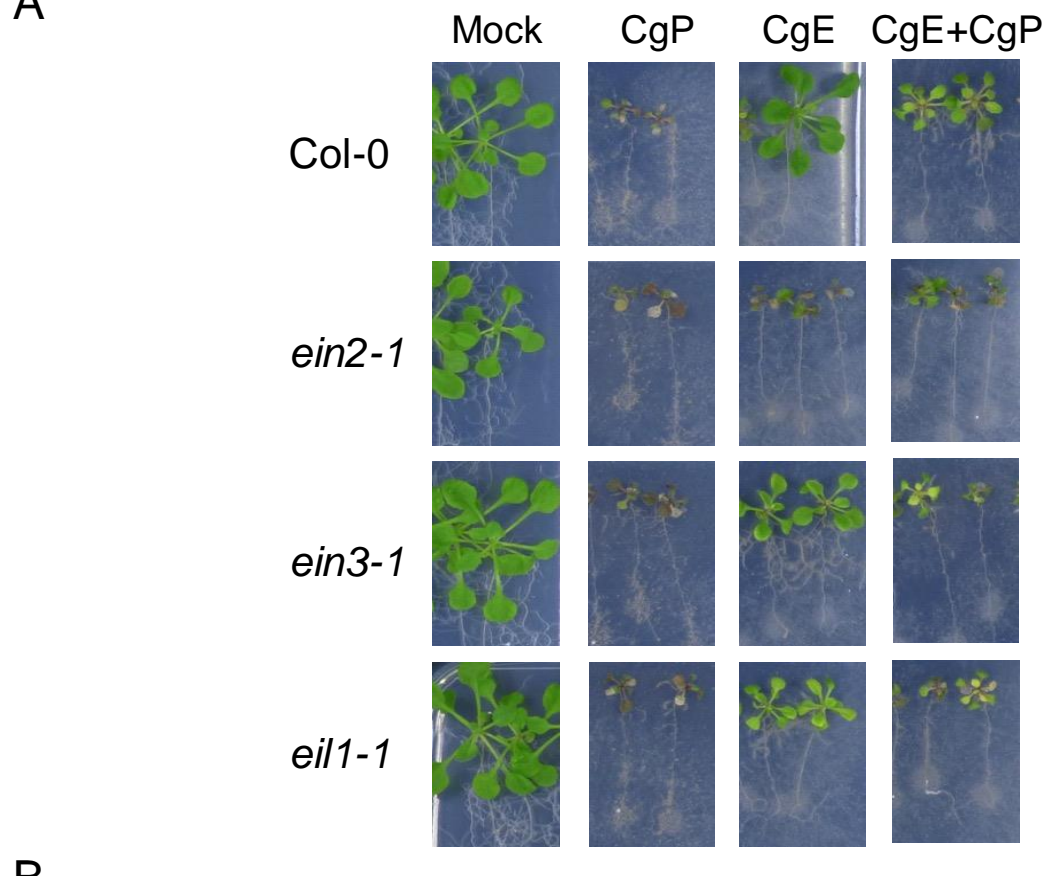

B

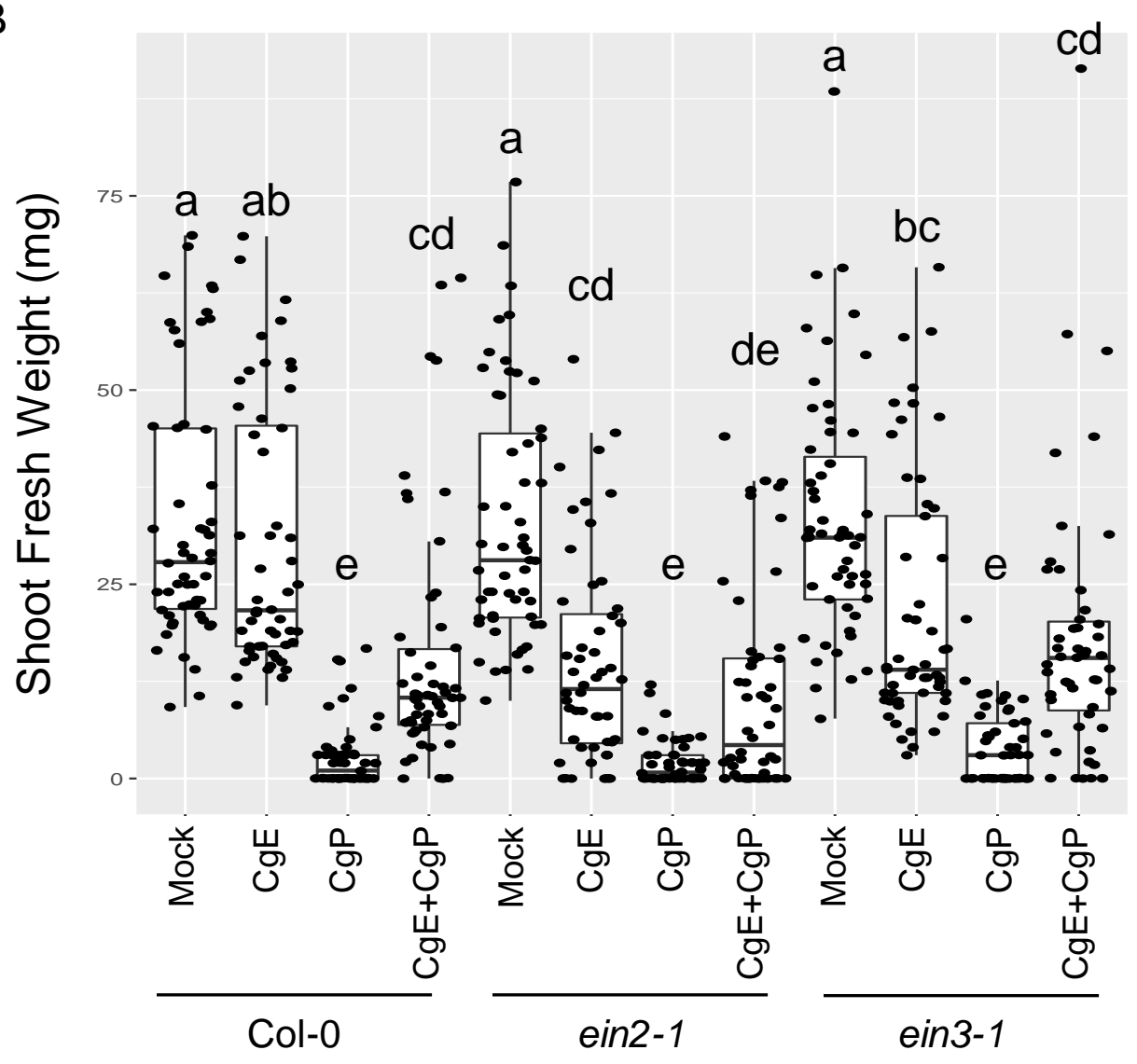

Fig. 4. Endophytic $\mathrm{CgE}$ colonization and host protection require plant ethylene signaling (A) Morphology of the plants treated with water, $\mathrm{CgE}, \mathrm{CgP}$ or co-inoculation of $\mathrm{CgE}$ with $\mathrm{CgP}$ $(\mathrm{CgE}+\mathrm{CgP})$ at $21 \mathrm{dpi}$ on 1/2 MS agar media. (B) Measurement of the shoot fresh weight of wildtype Arabidopsis and ET-related mutants (ein2-1, ein3) in the co-inoculation assay at 14 dpi. Each sample comprised around 20 shoots per experiment. The boxplot shows combined data from three independent experiments. Different letters indicate significantly different statistical groups (Tukey's HSD, $p<0.05$ ). 
A



B



Fig. 5. Endophytic $\mathrm{CgE}$ colonization and host protection require host tryptophan (Trp)-derived metabolites

(A) Scheme for Trp-derived metabolite pathways in Arabidopsis thaliana. Measurement of the shoot fresh weight of Arabidopsis thaliana Trp-pathway mutant plants treated with water, $\mathrm{CgE}$ and $\mathrm{CgP}$ or co-inoculated with $\mathrm{CgE}$ and $\mathrm{CgP}$ at $14 \mathrm{dpi}$. The boxplot shows combined data from three independent experiments. Different letters indicate significantly different statistical groups (Tukey's HSD, $p<0.05$ ). 

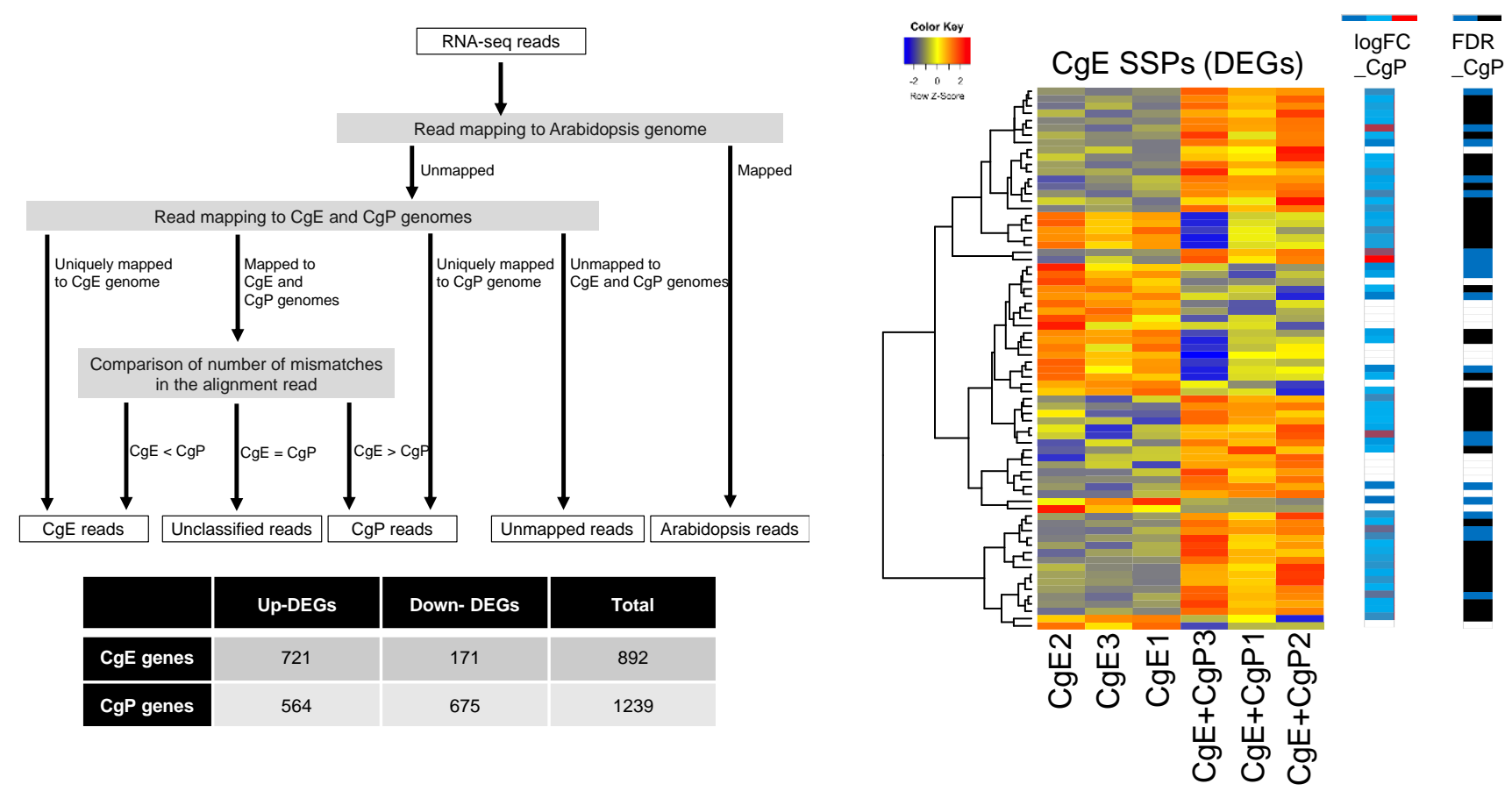

C

D
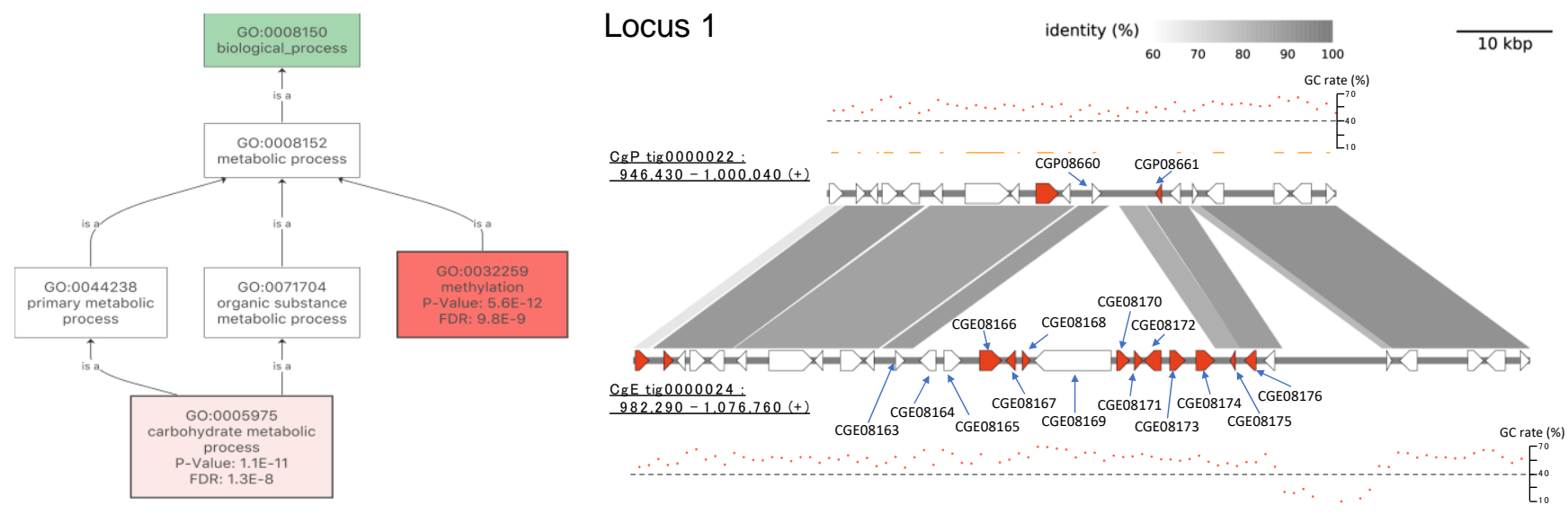

Fig. 6. Transcriptome analysis of fungi detects large gene expression changes in co-inoculated samples

(A) Schematic diagram of the classification of RNA-seq reads from co-inoculated samples. The table represents a number of $\mathrm{CgE}$ or $\mathrm{CgP}$ genes specifically upregulated or downregulated in co-inoculated samples compared with that in the corresponding single-inoculated samples at 3 dpi. DEGs = differentially expressed genes. (B) Transcript profiling of $74 \mathrm{CgE} \mathrm{SSP} \mathrm{DEGs}\left(\left|\log _{2} \mathrm{FC}\right| \geqslant 1, \mathrm{FDR}<0.05\right)$ between $\mathrm{CgE}$-colonized versus (vs) $\mathrm{CgE}+\mathrm{CgP}$-colonized roots. Overrepresented (yellow to red) and underrepresented transcripts (yellow to blue) are shown as $\log _{10}$ (read count +1 ). LogFC_CgP represents logFC (CgP-colonized vs $\mathrm{CgE}+\mathrm{CgP}$-colonized roots) of the corresponding $\mathrm{CgP}$ genes (Blue to Red). White represents the absence of obvious homologs in CgP (Similarity < 90\%). FDR_CgP represents whether the expression levels of the corresponding $\mathrm{CgP}$ genes between $\mathrm{CgP}$-colonized and $\mathrm{CgE}+\mathrm{CgP}$-colonized roots are significant (Blue: FDR < 0.05, Black: $\mathrm{p}>0.05$, White: no homologs in $\mathrm{CgP}$ (Similarity < 90\%). (C) Results of Gene ontology (GO) analysis using $721 \mathrm{CgE}$ up-regulated DEGs in the co-inoculated samples. The enriched GO terms of biological process were shown. (D) The expression profiles of $\mathrm{CgE}$ genes located in the secondary metabolism cluster 25. The genomic sequences surrounding the secondary metabolism cluster 25 were extracted from the genome assembly of each allele in $\mathrm{CgE}$ and $\mathrm{CgP}$. Vertical bars connecting adjacent genomic structures indicate BLAST hit blocks in the comparison between the two adjacent genomic scaffolds. Polygons indicate predicted genes. The red represents significantly higher expression in the co-inoculated samples compared to its alone $\left(\log _{2} \mathrm{FC}>1, \mathrm{FDR}<0.05\right)$. GC rate indicates $\mathrm{GC}$ content per $1 \mathrm{~kb}$ window. 